\title{
Infrared Spectra of Eighteen Halogen-Substituted Methanes
}

\author{
Earle K. Plyler and W. S. Benedict
}

\begin{abstract}
In order to study how the vibrational frequencies of methane vary with halogen substitutions, the infrared spectra of the following halogen-substituted methanes have been studied with a prism spectrometer between 2 and 38 microns: Carbon tetrafluoride, chlorotrifluoromethane, dichlorodifluoromethane, trichlorofluoromethane, carbon tetrachloride, trichlorobromomethane, dichlorodibromomethane, fluoroform, difluorochloromethane, dichlorofluoromethane, chloroform, dichlorobromomethane, chlorodibromomethane, bromoform, iodoform, methylene fluoride, methylene chloride, and methylene iodide. Spectra are presented as obtained at room temperature in the vapor and liquid states, and in a few cases in solution. The bands are interpreted as fundamentals, overtones, combination, and difference bands. Tables and graphs display the regularities existing among the various fundamentals of these molecules and other halogen-substituted methanes. Force-constant calculations, using an approximate potential function with general constants transferred from one molecule to another are reported.
\end{abstract}

\section{Introduction}

The infrared absorption spectra of various halogen derivatives of methane have been studied by several investigators in various regions of the spectrum, and under varying degrees of dispersion [1]. ${ }^{1}$ The Raman spectra of these compounds have also been extensively studied [2]. The gradual improvement in infrared techniques, in particular the development of prism spectrographs using thallium bromide-iodide (KRS-5) as the dispersing medium, and the increasing practical importance of the fluorine-containing compounds (Freons) have made it seem worth while to carry out a comprehensive reinvestigation of these compounds over the wide spectral range of 2 to $38 \mu$, using prism spectrographs of various dispersing intervals, so that good dispersion is obtained throughout the entire region. Compounds were studied both in the vapor and liquid states, using in many cases comparatively thick cells in order to develop bands of low intensity.

A listing of the compounds studied, together with their boiling points and the phases in which they were observed, is given in table 1. Representatives of all types of halogen-substituted methanes except the methyl halides, which have been extensively studied under high resolution, were included.

Since the spectra were recorded with prism spectrographs, it was not possible to resolve the rotational fine-structure.

It should be possible to account for the observed absorption bands in terms of transitions among the various vibrational states of the molecules. 'The bands will be of three types: fundamentals, in which transition occurs from the ground vibrational level to a state in which one mode of vibration is singly excited; overtones and combinations, from the ground level to states in which there are two or three quanta of vibrational energy in one or more modes; and difference bands, in which transition is from one singly excited vibrational mode to other higher vibrational levels. In general the fundamentals will be

\footnotetext{
Figures in brackets indicate the literature references at the end of this paper.
}

the most intense bands, although in certain of the molecules, because of symmetry considerations, the intensity in the vapor phase should become zero. The combinations and overtones, lying at shorter wavelengths than the fundamentals, will be weaker, and the difference bands, which, in general, will be at longer wavelengths, will be very weak, except in the heavier molecules, where the fundamentals will be of such low energy that appreciable percentages of the molecules will be in excited states at room temperature. The following sections describe the experimental method, present the results, and discuss further the vibrational assignments.

\section{Experimental Methods and Results}

The infrared absorption spectra of 18 substituted methanes have been measured in the region 2 to $38 \mu$. They were measured in the liquid state, at room temperature whenever possible, and also several of the compounds, as listed in table 1 , have been measured in both the liquid and vapor state. The eight Freons have been measured as gases, except trichlorofluoromethane, in the $23-$ to $38-\mu$ region. In order to observe the bands of low intensity in liquids, a cell with a thickness of $1.6 \mathrm{~mm}$ was used. For the intense bands a film of liquid between windows constituted the cell. In such cases the cell thickness was of the order of $0.01 \mathrm{~mm}$. For the measurement of the gases and vapors the cell thickness varied from $5 \mathrm{~cm}$ to $1 \mathrm{~m}$. The pressure of the vapors was that of the saturated vapor at room temperature (approximately $25^{\circ} \mathrm{C}$ ). For the Freons the pressure was atmospheric or less for the different cell lengths. The liquid-cell thickness, gas-cell length, and gas pressure are given on graphs of the results. The spectra were recorded on a Perkin-Elmer spectrometer.

In order to cover the region from 2 to $38 \mu$ with good resolution, prisms of lithium fluoride, sodium chloride, potassium bromide, and thallium bromideiodide were used. Sharp bands could be measured 
with an accuracy of $5 \mathrm{~cm}^{-1}$ or less for the entire region. The general method of measurement was the same as that used in previous studies [3].

The eight fluorinated methane derivatives were supplied by W. S. Murray, of Kinetic Chemicals, Wilmington, Del. They were all above 99 percent in purity, except carbon tetrafluoride, whose spectrum showed one band that could not be accounted for by combination or overtone bands. It appeared in the same position as an intense band in chlorotrifluoromethane at about $9 \mu$. The carbon tetrafluoride gas was tested for purity by F. L. Mohler on the mass spectrometer and found to be 98.2 percent pure. The Michigan Chemical Co. supplied the chlorodibromomethane, dichlorobromomethane, trichlorobromomethane, and dichlorodibromomethane, and some of the others were from Dow Chemical Co. The bromoform and chloroform were obtained from Eastman Kodak Co. and were purified by W. Harold Smith, of the Organic Chemistry Section. After the first purification, the chloroform showed a band in the 3.4- $\mu$ region in addition to the intense band at $3.3 \mu$. After the second purification, the band at $3.4 \mu$ disappeared.

The results are presented as graphs of percentage absorption against wavelength and wave number in figures 1 to 12 , inclusive. The conditions of measurement are stated on each graph. In general, the spectra are presented in groups of five molecules of similar structure, and the spectrum is divided into two portions, 2 to $15 \mu$, corresponding to measurements with sodium chloride and lithium fluoride prisms, and 15 to $38 \mu$, corresponding to measurements with potassium bromide and thallium bromideiodide prisms.

Numerical values of the positions of maximum absorption that may be identified as band centers, and further data pertaining to their interpretation, are presented in tables 2 to 6 , inclusive. These include the band centers in wave numbers; the approximate intensity, characterized as weak, strong, etc. (for a more quantitative estimate, the graphs should be consulted); the vibrational transition; and the symmetry of the transition. In addition to bands that appear in the infrared spectrum, there are given in parentheses the fundamental frequencies that do not appear, either because they fall outside the range of wavelengths studied, or because they have zero or very weak intensities. These frequencies are obtained either from the Raman effect or from combination and difference bands. The values given refer in general to the liquid state, except for compounds that are gaseous at room temperature. Cases where there is a pronounced difference between the two states will be mentioned in the discussion.

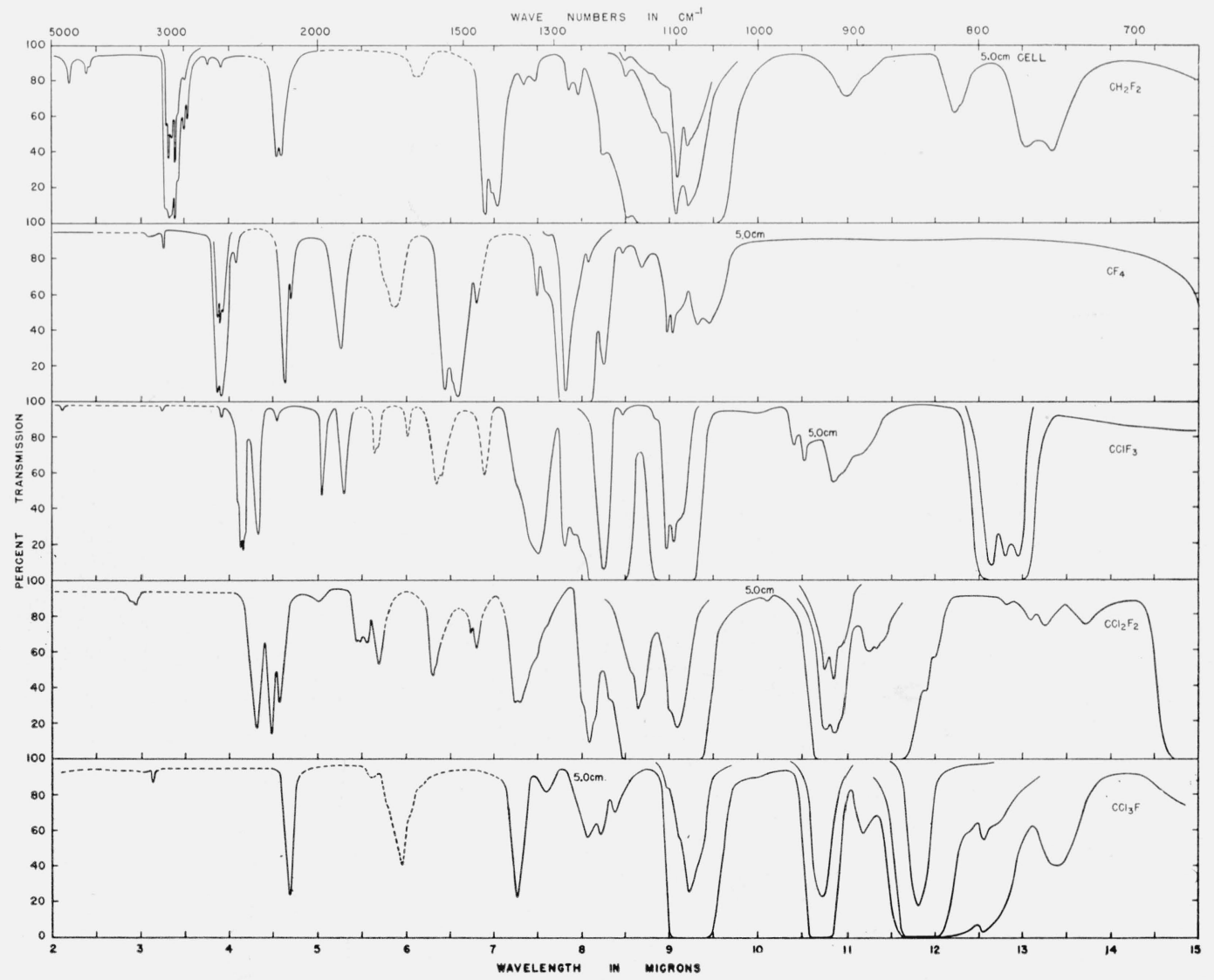

Figure 1. Infrared spectra of gaseous $\mathrm{CH}_{2} \mathrm{~F}_{2}, \mathrm{CF}_{4}, \mathrm{CClF}_{3}, \mathrm{CCl}_{2} \mathrm{~F}_{2}$, and $\mathrm{CCl}_{3} \mathrm{~F}$ from 2 to $15 \mu$. 


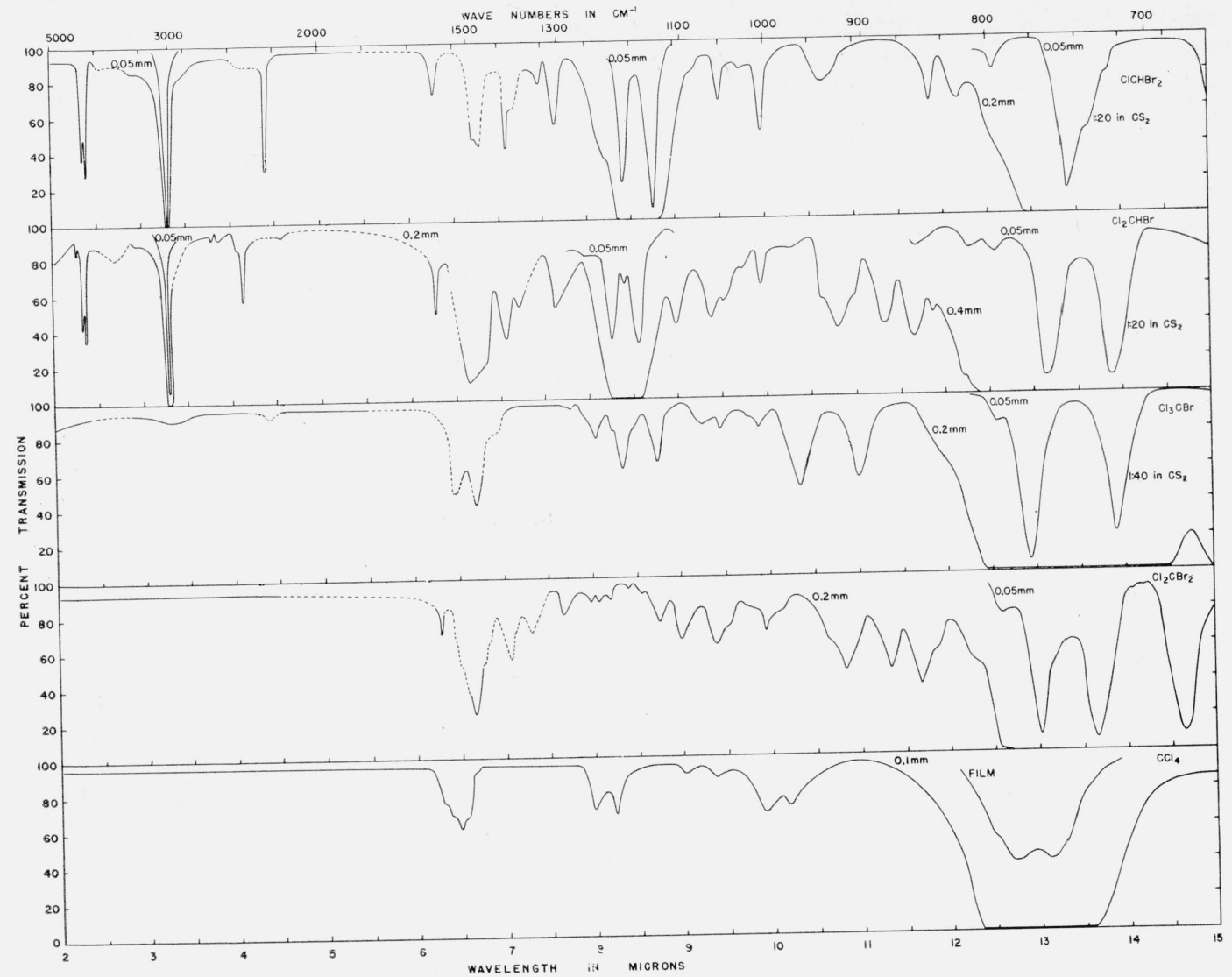

Figure 2. Infrared spectra of $\mathrm{CHBr}_{2} \mathrm{Cl}$, $\mathrm{CHBrCl}_{2}, \quad \mathrm{CBrCl}_{3}$, $\mathrm{CBr}_{2} \mathrm{Cl}_{2}$, and $\mathrm{CCl}_{4}$ in the liauid state.

Some strong bands near $14 \mu$ were measured in solution.

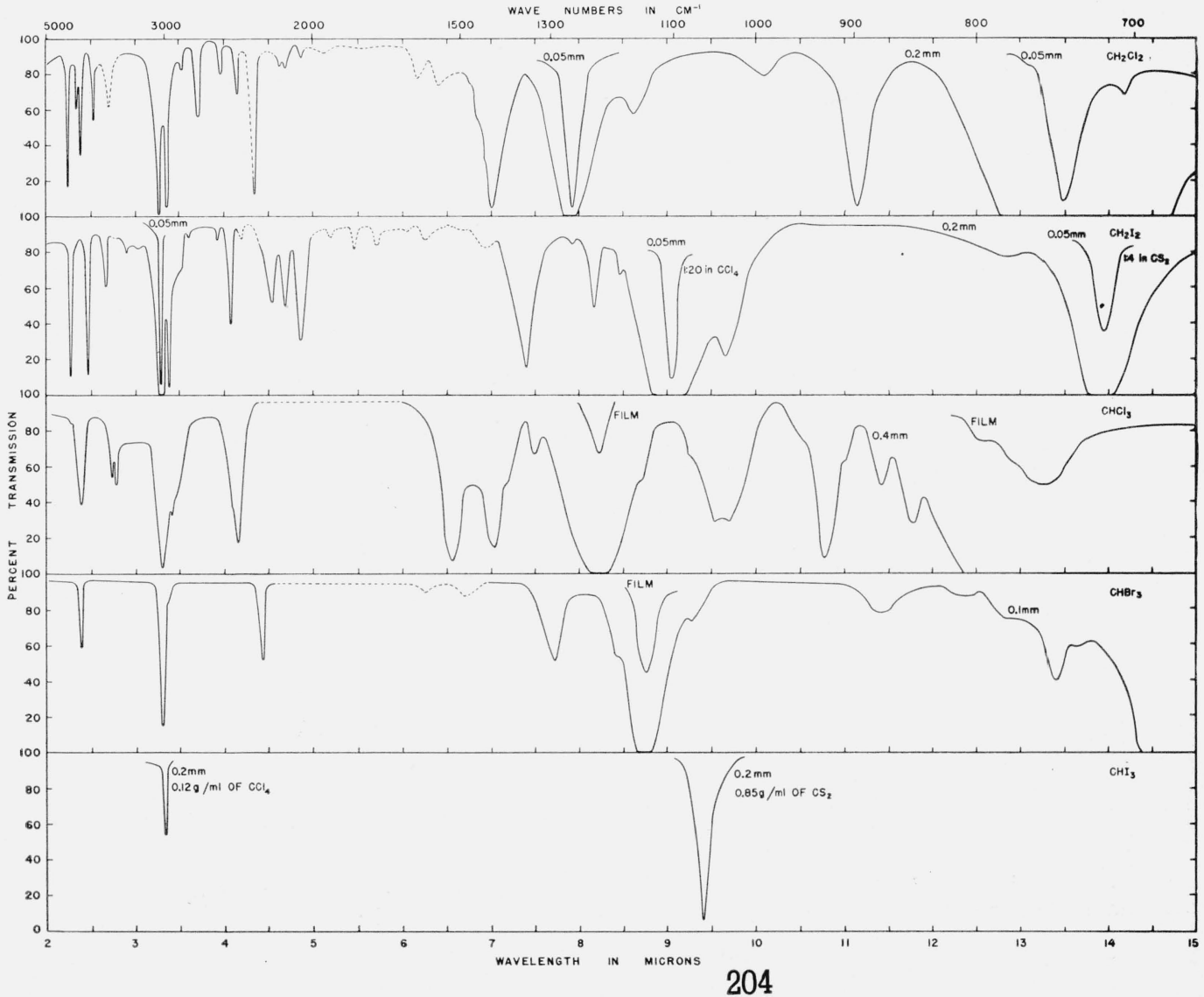

Figure 3. Infrared spectra of $\mathrm{CH}_{2} \mathrm{Cl}_{2}$, $\mathrm{CH}_{2} \mathrm{I}_{2}, \mathrm{CHCl}_{3}$, $\mathrm{CHBr}_{3}$, in the liquid state and $\mathrm{CHI}_{3}$ in solution from 2 to $15 \mu$. 


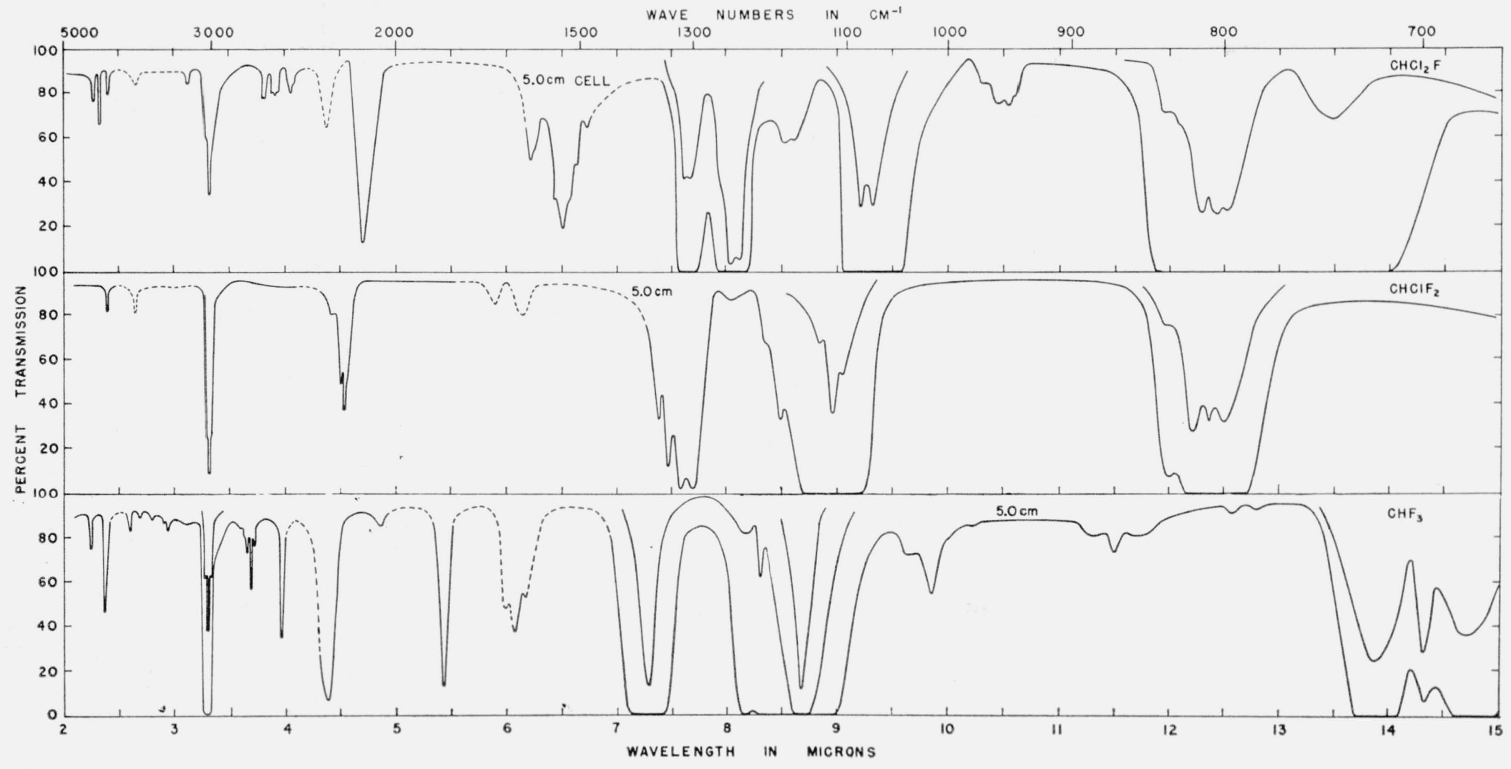

Figure 4. Infrared spectra of gaseous $\mathrm{CHCl}_{2} \mathrm{~F}, \mathrm{CHClF}_{2}$, and $\mathrm{CHF}_{3}$ from 2 to $15 \mu$.

Pressure is atmospheric. Insert graphs are for pressures less than atmospheric.

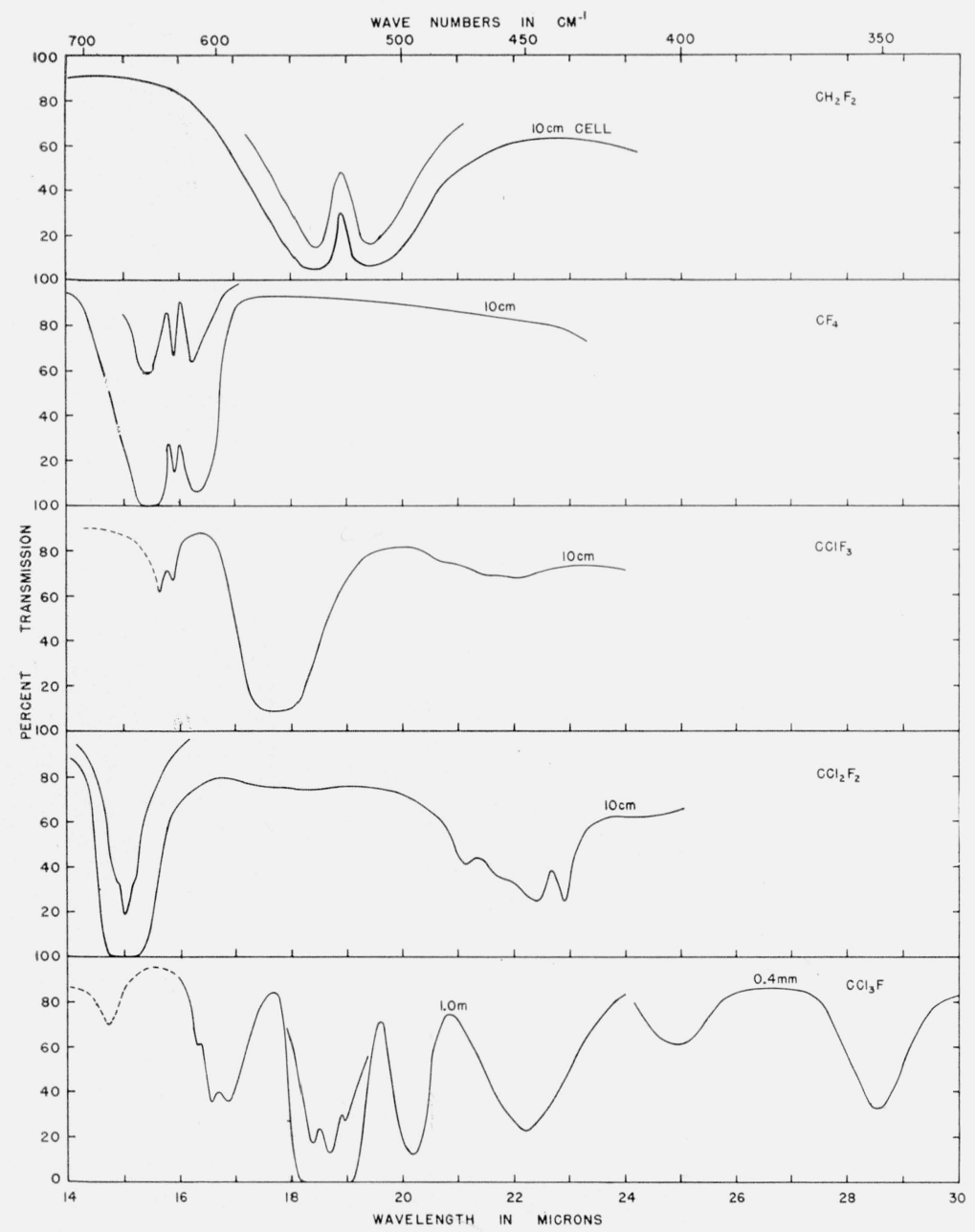

Figure 5. Infrared spectra of gaseous $\mathrm{CH}_{2} \mathrm{~F}_{2}, \mathrm{CF}_{4}, \mathrm{CClF}_{3}, \mathrm{CCl}_{2} \mathrm{~F}_{2}$, and $\mathrm{CCl}_{3} \mathrm{~F}$ from 14 to $30 \mu$.

Insert graphs are for pressures less than atmospheric. For $\mathrm{CCl}_{3} \mathrm{~F}$ the $0.4-\mathrm{mm}$ cell refers to the liquid state. 


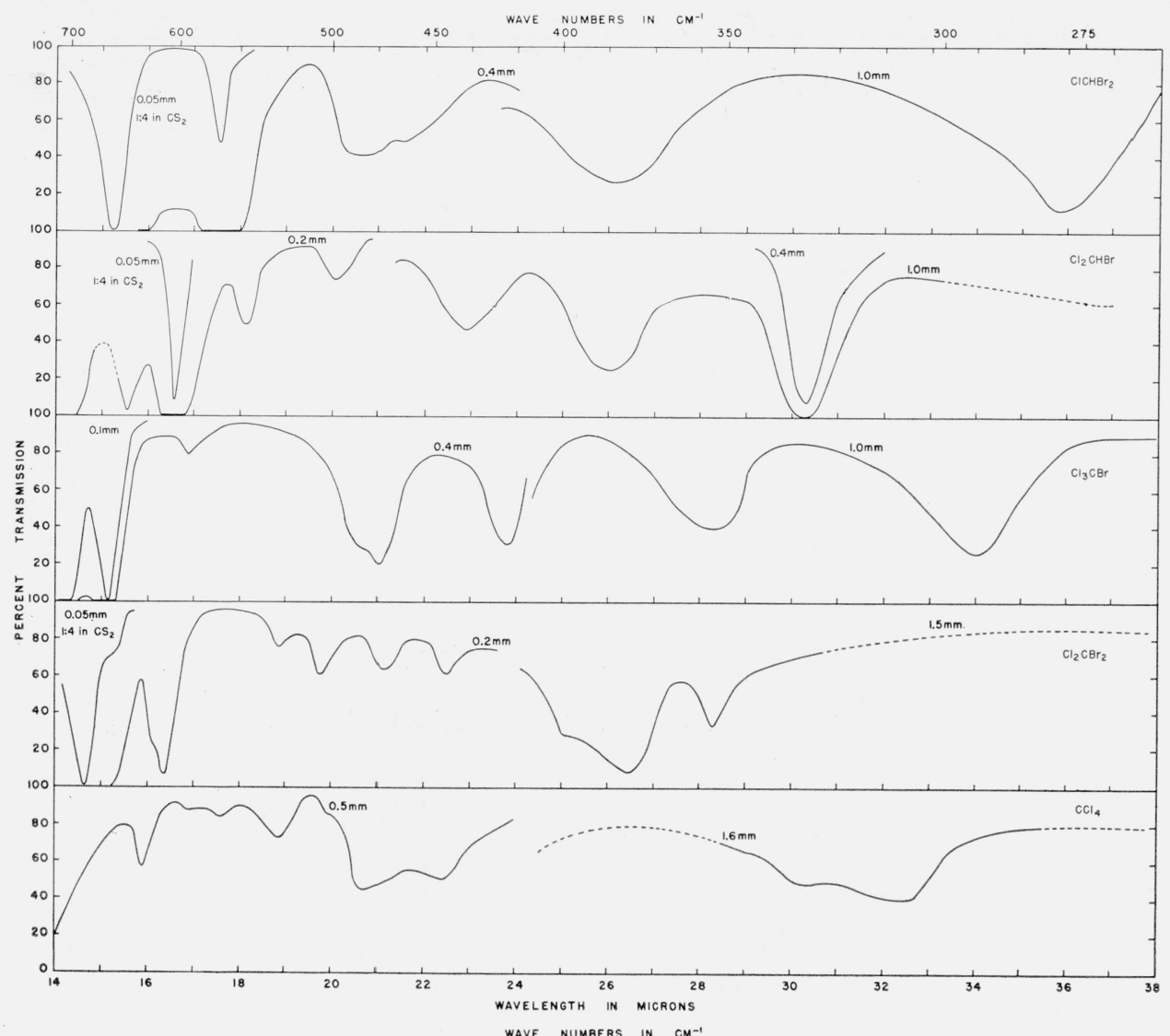

Figure 6. Infrared spectra of $\mathrm{CHBr}_{2} \mathrm{Cl}, \mathrm{CHBrCl}_{2}$, $\mathrm{CBrCl}_{3}, \mathrm{CBr}_{2} \mathrm{Cl}_{2}$, and $\mathrm{CCl}_{4}$ from 14 to $38 \mu$ in the liquid state.

The stronger bands near $16 \mu$ were measured in solution.

Figure 7. Infrared spectra of $\mathrm{CH}_{2} \mathrm{Cl}_{2}, \mathrm{CH}_{2} \mathrm{I}_{2}, \mathrm{CHCl}_{3}$, $\mathrm{CHBr}_{3}$, in the liquid state, and $\mathrm{CHI}_{3}$ in solution from 14 to $38 \mu$. 


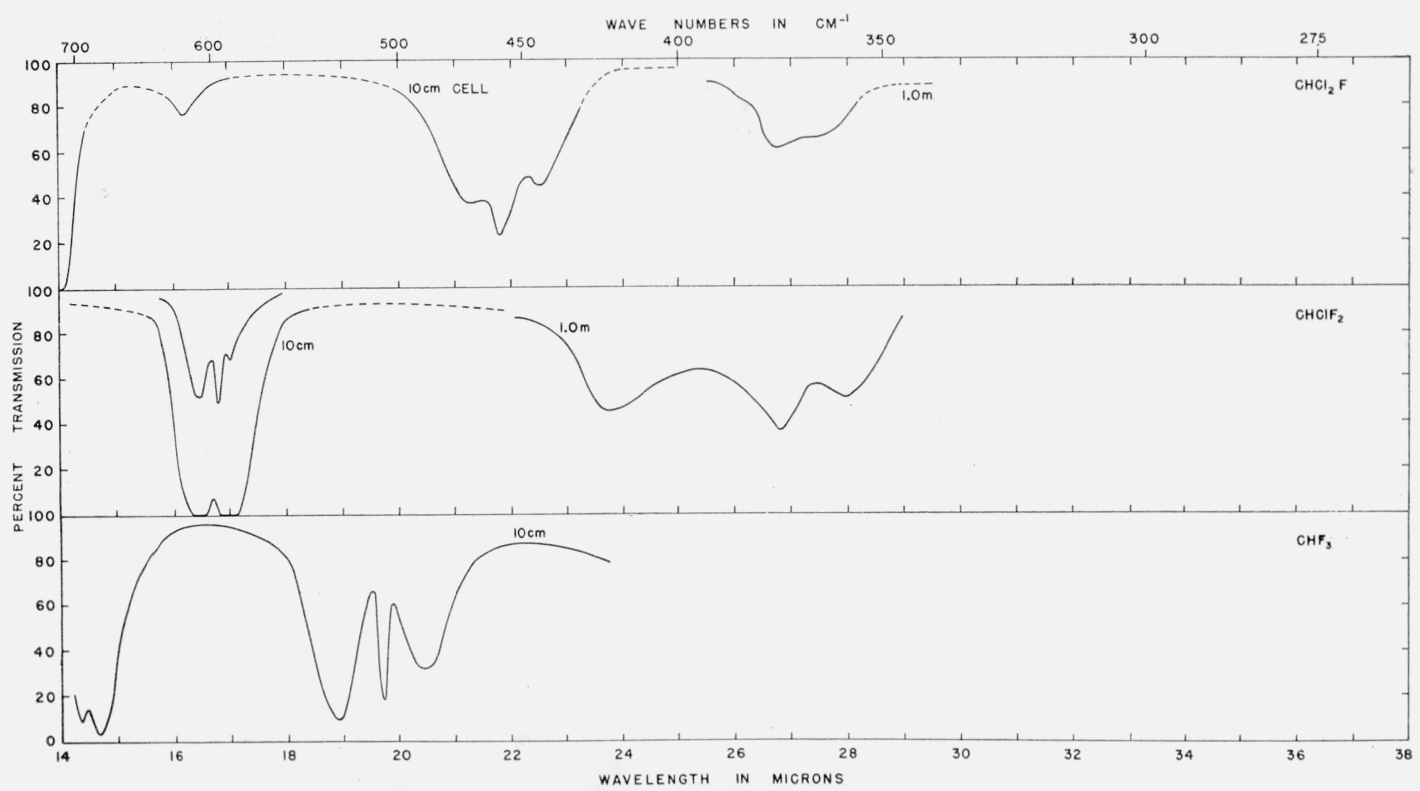

FIgure 8. Infrared spectra of gaseous $\mathrm{CHCl}_{2} \mathrm{~F}, \mathrm{CHClF}_{2}$, and $\mathrm{CHF}_{3}$ from 14 to $38 \mu$.

Pressure is atmospheric. Insert graph is for a pressure less than atmospheric.

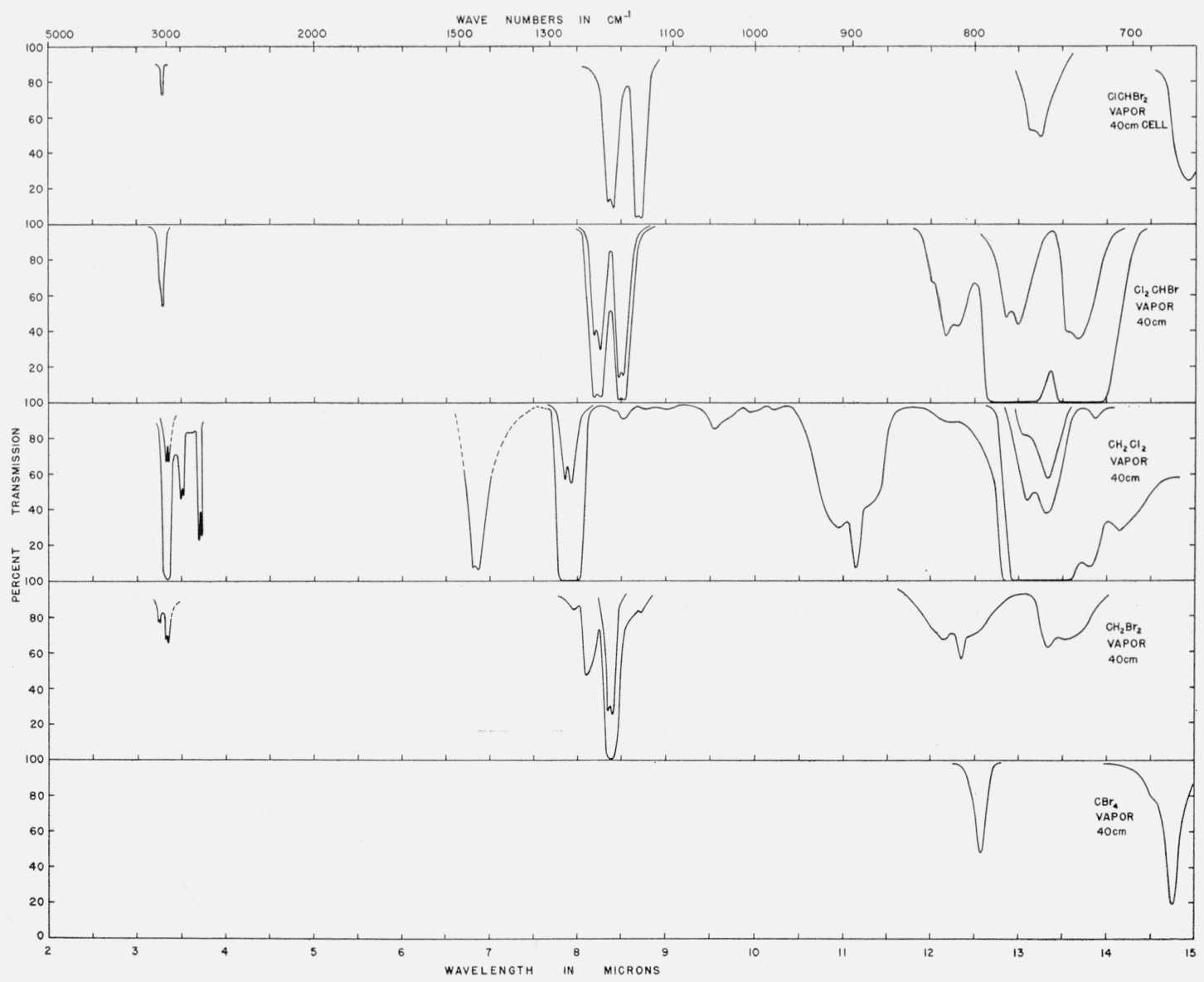

FIGURE 9. Infrared spectra of vapors at saturation pressures at room temperature of $\mathrm{CHBr}_{2} \mathrm{Cl}, \mathrm{CHBrCl}_{2}$, $\mathrm{CH}_{2} \mathrm{Cl}_{2}, \mathrm{CH}_{2} \mathrm{Br}_{2}$, and $\mathrm{CBr}_{4}$, from 2 to $15 \mu$.

The insert curves are for reduced vapor pressures. 


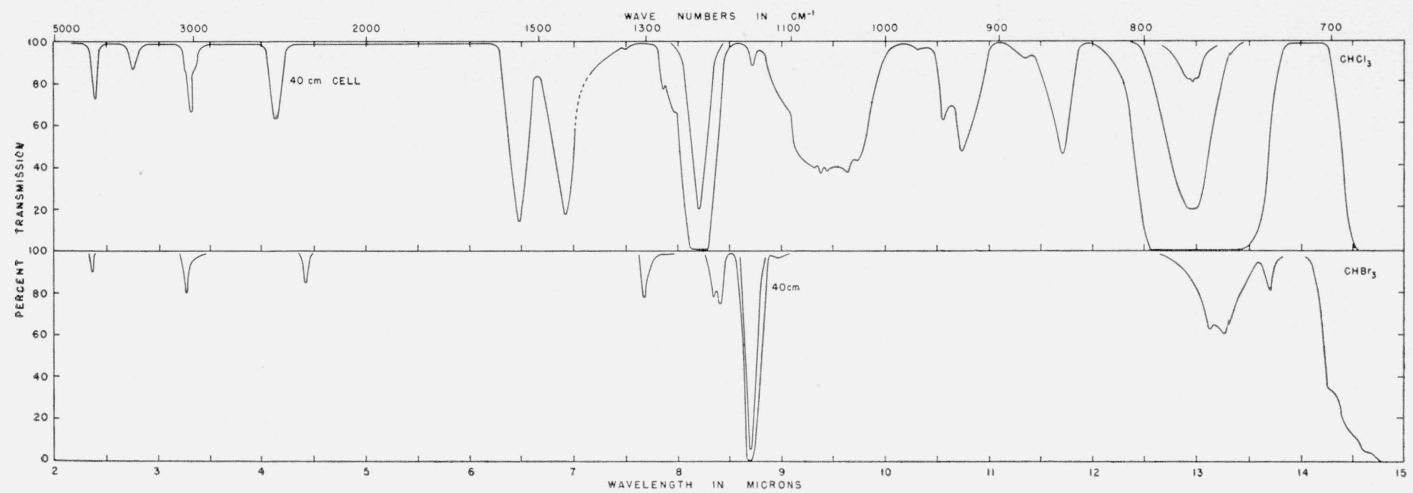

FIGURE $10 . \quad I n$. frared spectra of vapors saturated at room temperature of $\mathrm{CHCl}_{3}$, and $\mathrm{CHBr}_{3}$ from 2 to $15 \mu$.

The inserts are for re. duced pressure.

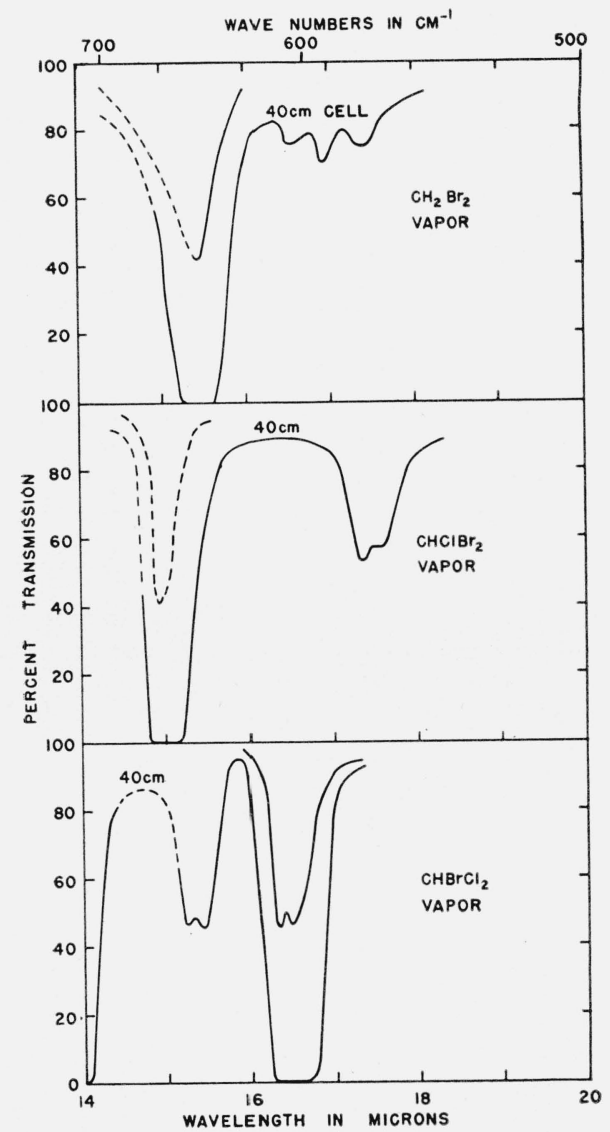

Figure 11. Infrared spectra of saturated vapors of $\mathrm{CH}_{2} \mathrm{Br}_{2}, \mathrm{CHBr} 2 \mathrm{Cl}$, and $\mathrm{CHBrCl}_{2}$ from 14 to $20 \mu$.

Insert curves are for reduced pressure.

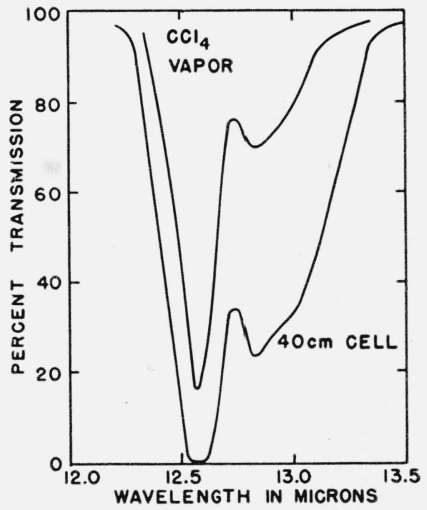

FIGURE 12. Infrared absorption spectra of saturated vapor of $\mathrm{CCl}_{4}$ at room temperature.

Insert curve is for reduced pressure.

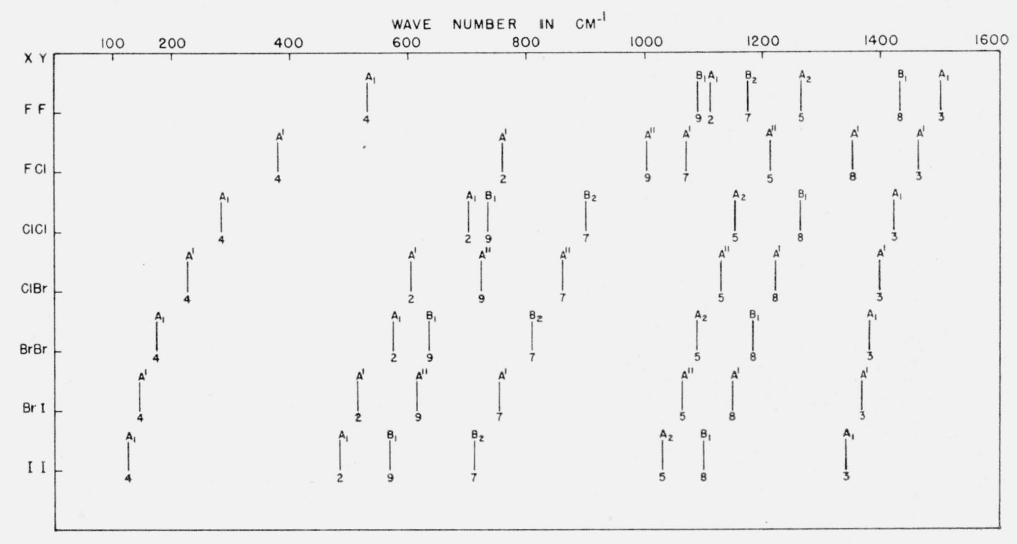

VIBRATIONS OF $\mathrm{CH}_{2} X Y$ MOLECULES

Figure 13. Comparison of the positions of the fundamental bands of molecules of type $\mathrm{CH}_{2} \mathrm{XY}$. 


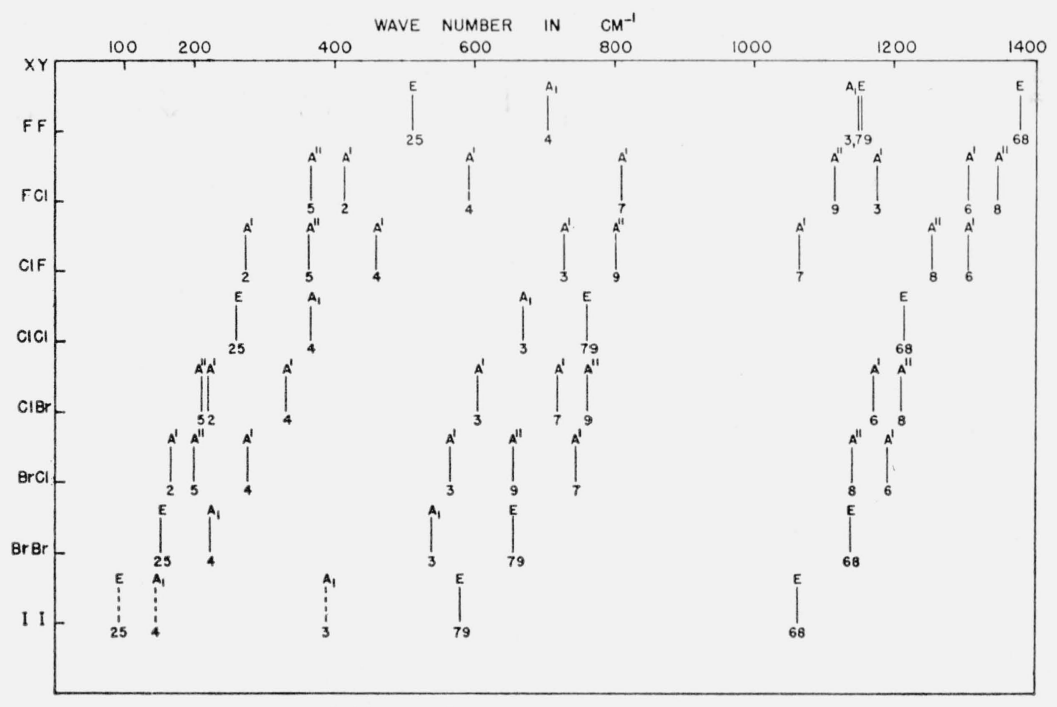

VIBRATIONS OF $\mathrm{CHX}_{2} Y$ MOLECULES

Figure 14. Comparison of the positions of the fundamental bands of molecules of type $\mathrm{CHX}_{2} \mathrm{Y}$.

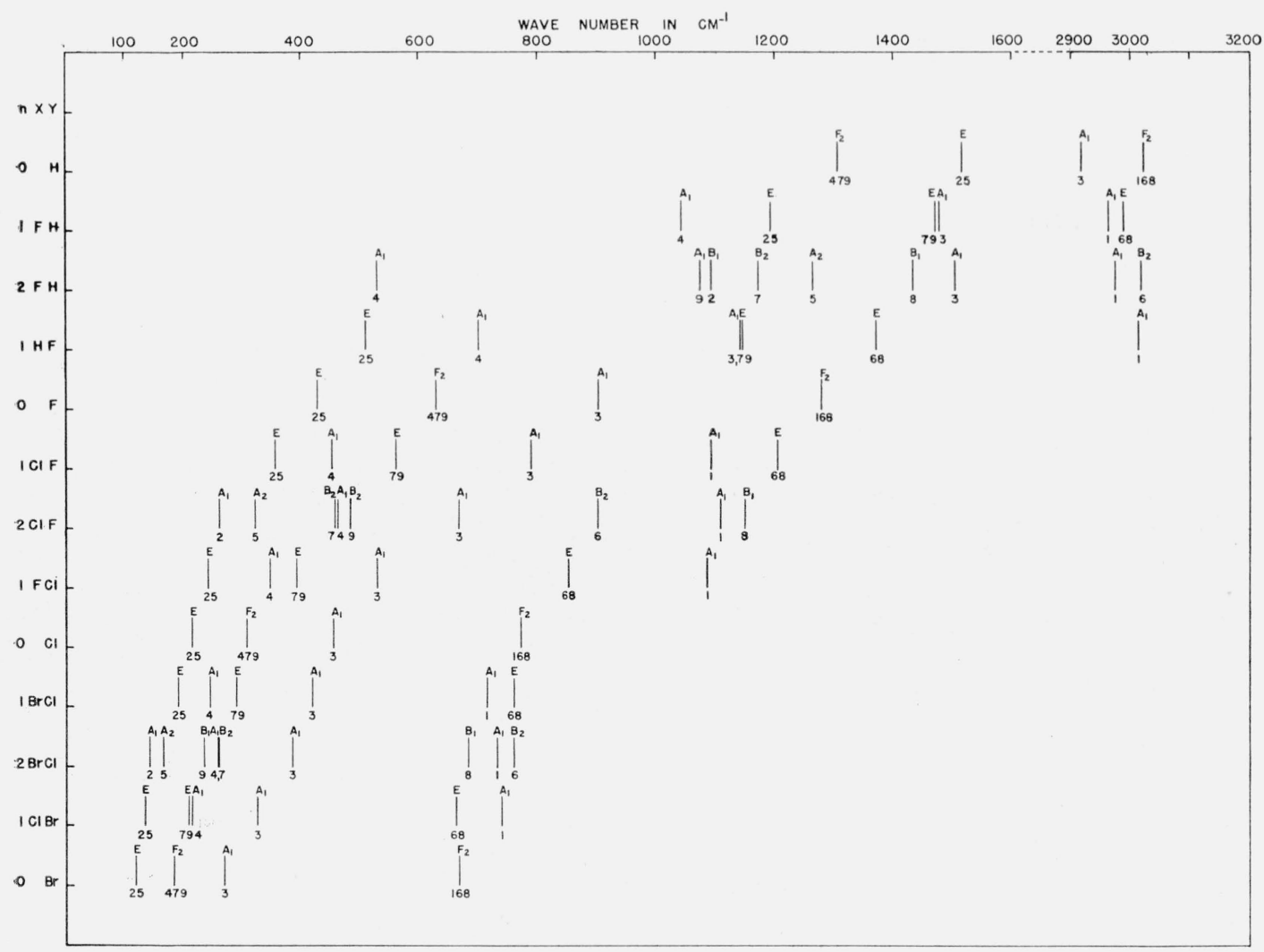

VIBRATIONS OF $C X_{n} Y_{4-n}$ MOLECULES

Figure 15. Comparison of the positions of the fundamental bands of molecules of the type $\mathrm{CX}_{\mathrm{n}} \mathrm{Y}_{4-n}$. 
TABLE 1. Some physical properties of the compounds measured

\begin{tabular}{|c|c|c|c|c|c|}
\hline \multirow[b]{2}{*}{ Compound } & \multirow[b]{2}{*}{ Formula } & \multirow{2}{*}{$\begin{array}{l}\text { Boiling } \\
\text { point a }\end{array}$} & \multicolumn{3}{|c|}{ Observed as- } \\
\hline & & & Gas & $\begin{array}{l}\text { Liq- } \\
\text { uid }\end{array}$ & $\begin{array}{l}\text { Solu- } \\
\text { tion }\end{array}$ \\
\hline $\begin{array}{l}\text { Carbon tetrafluoride (Freon 14) } \\
\text { Chlorotrifluoromethane (Freon 13) } \\
\text { Dichlorodifluoromethane (Freon } \\
\text { 12). } \\
\text { Trichlorofluoromethane (Freon 11) } \\
\text { Carbon tetrachloride } \\
\text { Trichlorobromomethane } \\
\text { Dichlorodibromomethane } \\
\text { Fluoroform (Freon 23) } \\
\text { Difluorochloromethane (Freon 22) } \\
\text { Dichlorofluoromethane (Freon 21) } \\
\text { Chloroform } \\
\text { Dichlorobromomethane } \\
\text { Chlorodibromomethane } \\
\text { Bromoform } \\
\text { Iodoform } \\
\text { Methylene fluoride (Freon 32) } \\
\text { Methylene chloride. } \\
\text { Methylene iodide. }\end{array}$ & $\begin{array}{l}\mathrm{CF}_{4} \\
\mathrm{CF}_{3} \mathrm{Cl}_{2} \\
\mathrm{CF}_{2} \mathrm{Cl}_{2} \\
\mathrm{CCl}_{3} \mathrm{~F} \\
\mathrm{CCl}_{4} \\
\mathrm{CCl}_{3} \mathrm{Br} \\
\mathrm{CCl}_{2} \mathrm{Br}_{2} \\
\mathrm{CHF}_{3} \\
\mathrm{CH}_{2} \mathrm{Cl} \\
\mathrm{CHFCl}_{2} \\
\mathrm{CHCl}_{3} \\
\mathrm{CHCl}_{2} \mathrm{Br} \\
\mathrm{CHClBr}_{2} \\
\mathrm{CHBr}_{3} \\
\mathrm{CH}_{3} \\
\mathrm{CH}_{2} \mathrm{~F}_{2} \\
\mathrm{CH}_{2} \mathrm{Cl}_{2} \\
\mathrm{CH}_{2} \mathrm{I}_{2}\end{array}$ & 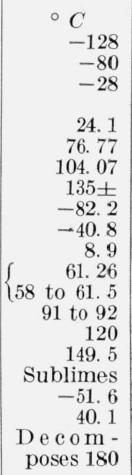 & $\left\{\begin{array}{l}x \\
x \\
x \\
x \\
x \\
x \\
x\end{array}\right.$ & $\begin{array}{l}x \\
\times \\
x \\
X\end{array}$ & $\begin{array}{l}\dot{x} \\
\times \\
\times \\
\times \\
\times\end{array}$ \\
\hline
\end{tabular}

a These values are principally from the Handbook of Physics and Chemistry.

\section{Discussion}

\subsection{The Fundamental Frequencies}

Each of the related methane derivatives reported in this paper has nine fundamental frequencies of vibration. The general theory of the vibrations of such molecules has been discussed in many places and need not be repeated here. It will suffice to recall that when there are atoms of different kinds joined to the carbon atom, the symmetry of the molecule is less than when all four linked atoms are alike, and that the particular degree of symmetry governs the degeneracy of the vibrations, the general form of the molecular motions, and the resulting activity in both the infrared and the Raman spectrum. The pertinent information derivable from such symmetry considerations is summarized in table 7. The symmetry class of overtones, combinations, and difference bands of the various fundamentals follow well-known rules and are given in tables 2 to 6 .

The numerical values of the fundamentals show regular variations when going from one molecule to another in which a single substituent has been altered. This is a qualitative consequence of the facts that in all these substituted methanes the forces between the central atom and a particular peripheral atom are to a first approximation independent of the other peripheral atoms, and that these forces vary regularly with the mass of the peripheral atom. A more quantitative discussion will be given later. The relative positions and groupings of the numerical values are also affected by the symmetry. The conventional system of numerical identification of the fundamentals is based on the symmetry. This system fails to display the regularities existing among molecules of different symmetry. Accordingly, in this paper, one purpose of which was to show the consistency of all the frequencies of a large number of related compounds of different symmetry, use is made of an unconventional numbering system. The system closely resembles the conventional one for molecules of the type $\mathrm{CH}_{2} \mathrm{X}_{2}$, where there are no degeneracies. For molecules of higher symmetry than $\mathrm{C}_{2 \mathrm{v}}$, where degeneracy occurs, the degenerate frequencies have been assigned two or three numbers, so that all nine frequencies may be identified. The relation between the numbers adopted here and those that would follow from application of the conventional rules is shown in table 8 . This table also collects in one place the numerical values of the fundamentals of all the molecules, the spectra of which are reported in this paper, as well as of related molecules that have recently been studied in this laboratory [4].

TABLE 2. The observed bands and their assignments of $\mathrm{CF}_{4}$, $\mathrm{CF}_{3} \mathrm{Cl}, \mathrm{CF}_{2} \mathrm{Cl}_{2}$, and $\mathrm{CFCl}_{3}$

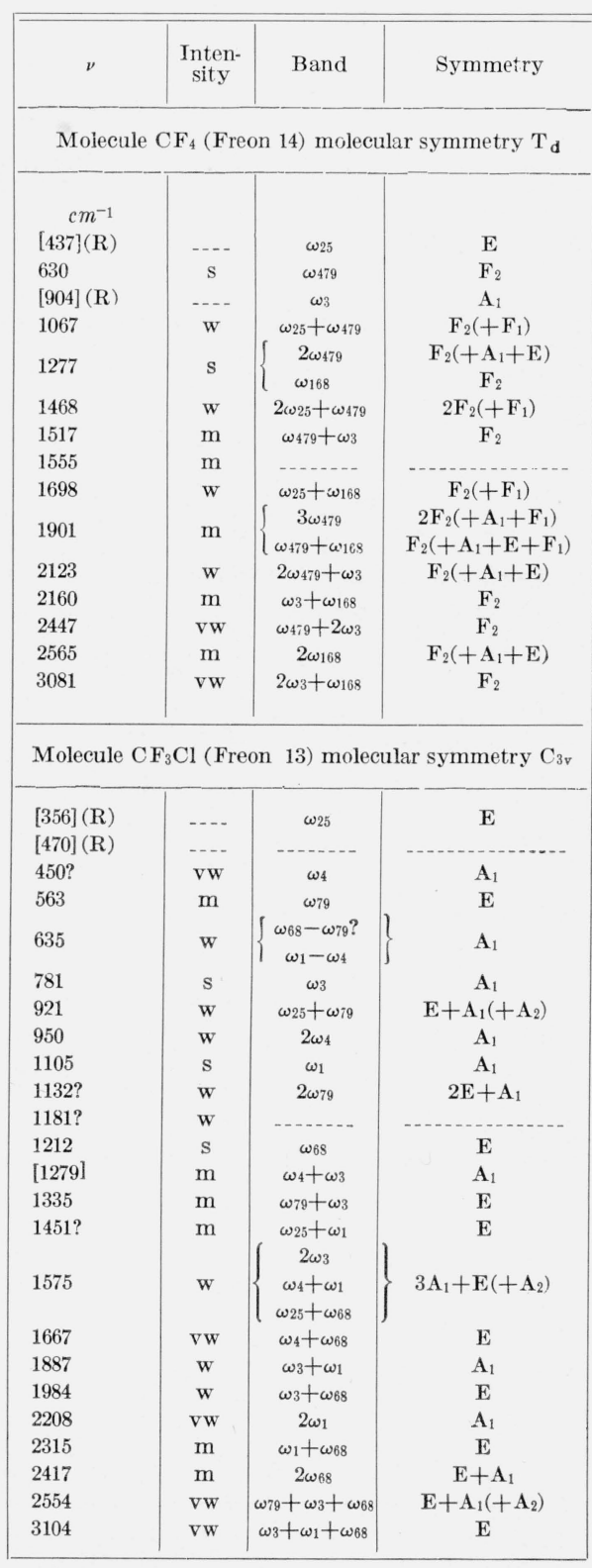


TABLE 2. The observed bands and their assignments of $\mathrm{CF}_{4}$, $\mathrm{CF}_{3} \mathrm{Cl}, \mathrm{CF}_{2} \mathrm{Cl}_{2}$, and $\mathrm{CFCl}_{3}-$ Continued

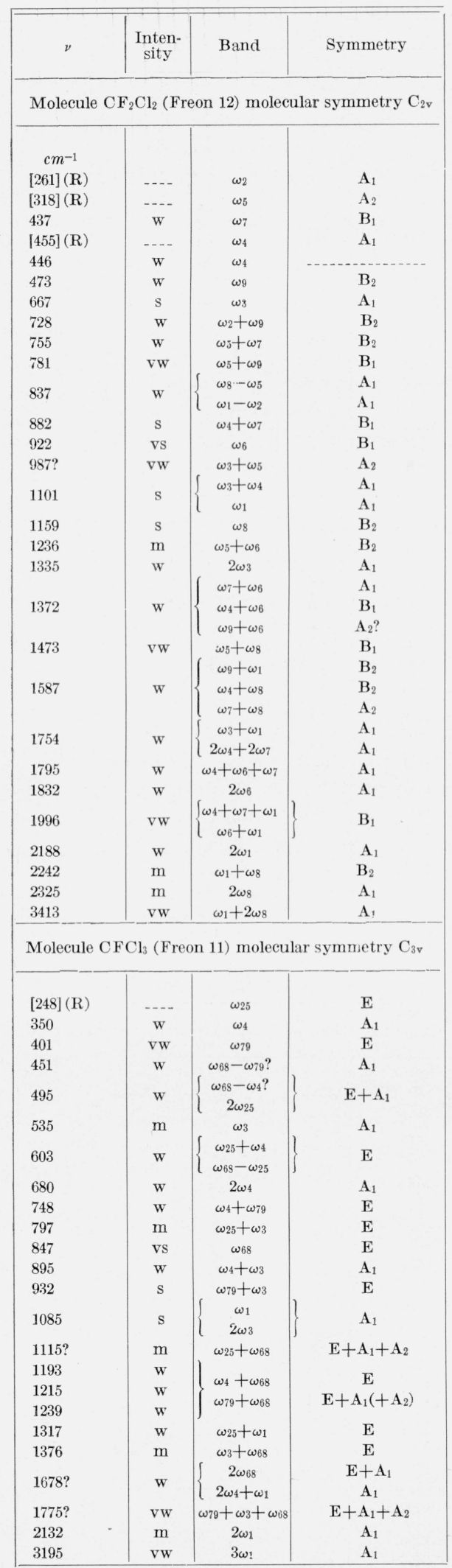

TABLE 3. The observed bands and their assignments of $\mathrm{CCl}_{4}$ $\mathrm{CCl}_{3} \mathrm{Br}$, and $\mathrm{CCl}_{2} \mathrm{Br}_{2}$

\begin{tabular}{|c|c|c|c|}
\hline$\nu$ & $\begin{array}{l}\text { Inten- } \\
\text { sity }\end{array}$ & Band & Symmetry \\
\hline \multicolumn{4}{|c|}{ Molecule $\mathrm{CCl}_{4}$ molecular symmetry $\mathrm{T}_{\mathrm{d}}$} \\
\hline $\begin{array}{l}\quad c m-1 \\
\quad[218](\mathrm{R}) \\
309 \\
319 \\
329 \\
446 \\
482 \\
{[458](\mathrm{R})} \\
501 \\
531 \\
569 \\
635 \\
762 \\
785 \\
982 \\
1006 \\
1068 \\
1107 \\
1218 \\
1253 \\
1529 \\
1546 \\
1575\end{array}$ & 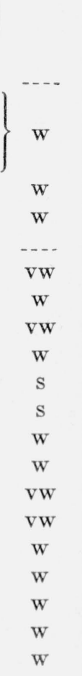 & 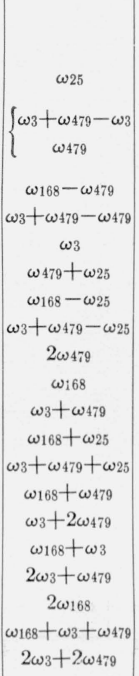 & $\begin{array}{c}(\mathrm{E}) \\
\mathrm{F}_{2} \\
\mathrm{~F}_{2} \\
\mathrm{~F}_{2} \\
\left(\mathrm{~A}_{1}\right) \\
\mathrm{F}_{2}\left(+\mathrm{F}_{1}\right) \\
\mathrm{F}_{2} \\
\mathrm{~F}_{2} \\
\mathrm{~F}_{2}\left(+\mathrm{E}+\mathrm{A}_{1}\right) \\
\mathrm{F}_{2} \\
\mathrm{~F}_{2} \\
\mathrm{~F}_{2}\left(+\mathrm{F}_{1}\right) \\
\mathrm{F}_{2}\left(+\mathrm{F}_{1}\right) \\
\mathrm{F}_{2}\left(+\mathrm{F}_{1}+\mathrm{E}+\mathrm{A}_{1}\right) \\
\mathrm{F}_{2}\left(+\mathrm{E}+\mathrm{A}_{1}\right) \\
\mathrm{F}_{2} \\
\mathrm{~F}_{2} \\
\mathrm{~F}_{2}\left(+\mathrm{E}+\mathrm{A}_{1}\right) \\
\mathrm{F}_{2}\left(+\mathrm{F}_{1}+\mathrm{E}+\mathrm{A}_{1}\right) \\
\mathrm{F}_{2}\left(+\mathrm{E}+\mathrm{A}_{1}\right)\end{array}$ \\
\hline \multicolumn{4}{|c|}{ Molecule $\mathrm{CCl}_{3} \mathrm{Br}$ molecular symmetry $\mathrm{C}_{3 \mathrm{v}}$} \\
\hline$[187](\mathrm{R})$ & - & $\omega_{25}$ & $\mathrm{E}$ \\
\hline$[243](\mathrm{R})$ & -... & $\omega 4$ & $\mathrm{~A}_{1}$ \\
\hline 294 & w & $\int \begin{array}{c}\omega_{79} \\
\omega_{68}-\omega_{3}\end{array}$ & $\begin{array}{l}\mathrm{E} \\
\mathrm{E}\end{array}$ \\
\hline $\begin{array}{l}353 \\
420\end{array}$ & $\mathrm{w}$ & $\left\{\begin{array}{c}2 \omega_{25} \\
\omega_{3}\end{array}\right.$ & $\begin{array}{c}\mathrm{E}+\mathrm{A}_{1} \\
\mathrm{~A}_{1}\end{array}$ \\
\hline 475 & $\mathrm{~m}$ & $\left\{\begin{array}{c}2 \omega_{4} \\
\omega_{1}-\omega_{4}\end{array}\right.$ & $\begin{array}{l}\mathbf{A}_{1} \\
\mathbf{A}_{1}\end{array}$ \\
\hline 490 & $\mathrm{w}$ & $\int \begin{array}{c}\omega_{79}+\omega_{25} \\
2 \omega 79\end{array}$ & $\begin{array}{c}\mathrm{E}+\mathrm{A}_{1}\left(+\mathrm{A}_{2}\right) \\
\mathrm{E}+\mathrm{A}_{1}\end{array}$ \\
\hline 592 & vw & $\left\{\begin{array}{c}\omega_{3}+\omega_{25} \\
\omega_{68}-\omega_{25}\end{array}\right.$ & $\begin{array}{l}\mathrm{E} \\
\mathrm{A}_{1}\end{array}$ \\
\hline 664 & $\mathrm{~s}$ & $\omega_{3}+\omega_{4}$ & $\mathbf{A}_{1}$ \\
\hline 719 & vs & $\omega_{1}$ & $\mathrm{~A}_{1}$ \\
\hline 773 & vs & $\omega_{68}$ & $\mathrm{E}$ \\
\hline 796 & $\mathrm{~m}$ & $2 \omega_{79}+\omega_{25}$ & $2 \mathrm{E}+\mathrm{A}_{1}\left(+\mathrm{A}_{2}\right)$ \\
\hline 908 & w & $\omega_{1}+\omega_{25}$ & $\mathbf{E}$ \\
\hline 966 & w & $\left\{\begin{array}{c}\omega_{1}+\omega_{4} \\
\omega_{68}+\omega_{25}\end{array}\right.$ & $\begin{array}{c}\mathrm{A}_{1} \\
\mathrm{E}+\mathrm{A}_{1}\left(+\mathrm{A}_{2}\right)\end{array}$ \\
\hline 1010 & vw & $\omega_{68}+\omega_{4}$ & E \\
\hline 1046 & $\mathrm{vW}$ & $\begin{array}{c}\omega_{1}+2 \omega_{25} \\
2 \omega_{3}+\omega_{4}\end{array}$ & $\begin{array}{c}\mathrm{E}+\mathrm{A}_{1} \\
\mathrm{~A}_{1}\end{array}$ \\
\hline $\begin{array}{l}1080 \\
1143\end{array}$ & $\begin{array}{l}\mathrm{vW} \\
\mathrm{W}\end{array}$ & $\begin{array}{c}2 \omega_{3}+\omega_{4} \\
\omega_{1}+\omega_{3}\end{array}$ & $\begin{array}{l}\mathbf{A}_{1} \\
\mathbf{A}_{1}\end{array}$ \\
\hline 1193 & $\mathrm{w}$ & $\omega_{68}+\omega_{3}$ & $\mathrm{E}$ \\
\hline 1242 & vw & $\omega_{1}+\omega_{68}-\omega_{4}$ & $\mathbf{E}$ \\
\hline 1440 & $\mathrm{w}$ & $2 \omega_{1}$ & $\mathbf{A}_{1}$ \\
\hline 1493 & $w$ & $\omega_{1}+\omega_{68}$ & $\mathrm{E}$ \\
\hline 1545 & $\mathrm{w}$ & $2 \omega_{68}$ & $\mathrm{E}+\mathrm{A}_{1}$ \\
\hline 2270 & $\mathrm{vw}$ & $2 \omega_{68}+\omega_{1}$ & $\mathrm{E}+\mathrm{A}_{1}$ \\
\hline
\end{tabular}


TABLE 3. The observed bands and their assignments of $\mathrm{CCl}_{4}$, $\mathrm{CCl}_{2} \mathrm{Br}$, and $\mathrm{CCl}_{2} \mathrm{Br}_{3}$-Continued

\begin{tabular}{|c|c|c|c|}
\hline$\nu$ & $\begin{array}{l}\text { Inten- } \\
\text { sity }\end{array}$ & Band & Symmetry \\
\hline \multicolumn{4}{|c|}{ Molecule $\mathrm{CCl}_{2} \mathrm{Br}_{2}$ molecular symmetry $\mathrm{C}_{2 \mathrm{v}}$} \\
\hline $\mathrm{cm}^{-1}$ & & & \\
\hline$[141](\mathrm{R})$ & $\ldots$ & $\omega_{z}$ & $\mathbf{A}_{1}$ \\
\hline$[164](\mathrm{R})$ & $-\ldots$ & $\omega_{5}$ & $\left(A_{2}\right)$ \\
\hline$[230](\mathrm{R})$ & .... & $\omega_{9}$ & $\mathrm{~B}_{1}$ \\
\hline$[252](\mathrm{R})$ & $\ldots$ & $\omega_{7}$ & $\mathrm{~B}_{2}$ \\
\hline$[252](\mathrm{R})$ & ...- & $\omega 4$ & $\mathrm{~A}_{1}$ \\
\hline 353 & $w$ & $\omega_{9}+\omega_{2}$ & $\mathrm{~B}_{1}$ \\
\hline 377 & $w$ & $\omega_{3}$ & $A_{1}$ \\
\hline 398 & $\mathrm{w}$ & $\begin{array}{l}\omega_{7}+\omega_{2} \\
\omega_{9}+\omega_{5}\end{array}$ & $\begin{array}{l}\mathrm{B}_{2} \\
\mathrm{~B}_{2}\end{array}$ \\
\hline 443 & $\mathrm{w}$ & $\left\{\begin{array}{c}2 \omega_{9} \\
\omega_{8}-\omega_{74}\end{array}\right.$ & $\begin{array}{c}\mathrm{A}_{1} \\
\mathrm{~B}_{1}\left(+\mathrm{A}_{2}\right)\end{array}$ \\
\hline 472 & $\mathrm{w}$ & $\omega_{9}+\omega_{74}$ & $\mathrm{~B}_{1}\left(+\mathrm{A}_{2}\right)$ \\
\hline 505 & $\mathrm{w}$ & $2 \omega_{74}$ & $2 \mathrm{~A}_{1}+\mathrm{B}_{2}$ \\
\hline 529 & $\mathrm{w}$ & $\begin{array}{l}\omega_{3}+\omega_{2} \\
\omega_{3}+\omega_{5}\end{array}$ & $\begin{array}{l}\mathbf{A}_{1} \\
\mathrm{~A}_{2}\end{array}$ \\
\hline 610 & $\mathrm{~m}$ & $\left\{\begin{array}{c}\omega_{3}+\omega_{974} \\
\omega_{6}-\omega_{2}\end{array}\right.$ & $\begin{array}{c}\mathrm{A}_{1}+\mathrm{B}_{1}+\mathrm{B}_{2} \\
\mathrm{~B}_{2}\end{array}$ \\
\hline 683 & vs & $\omega_{8}$ & $\mathrm{~B}_{1}$ \\
\hline 733 & vs & $\omega_{1}$ & $A_{1}$ \\
\hline 768 & vs & $\omega_{6}$ & $\mathrm{~B}_{2}$ \\
\hline 793 & $\mathrm{~m}$ & $\omega_{6}+\omega_{5}-\omega_{2}$ & $\mathrm{~B}_{1}$ \\
\hline 818 & $\mathrm{w}$ & $\omega_{8}+\omega_{2}$ & $\mathrm{~B}_{1}$ \\
\hline 844 & $\nabla W$ & $\omega_{8}+\omega_{5}$ & $\mathrm{~B}_{2}$ \\
\hline 858 & $\mathrm{w}$ & $\omega_{1}+\omega_{2}$ & $A_{1}$ \\
\hline 882 & $\mathrm{w}$ & $\begin{array}{l}\omega_{6}+\omega_{2} \\
\omega_{1}+\omega_{5}\end{array}$ & $\begin{array}{c}\mathrm{B}_{2} \\
\left(\mathrm{~A}_{2}\right)\end{array}$ \\
\hline 923 & $\mathrm{w}$ & $\left\{\begin{array}{l}\omega_{8}+\omega_{9} \\
\omega_{6}+\omega_{5}\end{array}\right.$ & $\begin{array}{l}\mathrm{A}_{1} \\
\mathrm{~B}_{1}\end{array}$ \\
\hline 939 & $v w$ & $\omega_{8}+\omega_{47}$ & $\mathrm{~B}_{1}\left(+\mathrm{A}_{2}\right)$ \\
\hline 991 & $v w$ & $\omega_{1}+\omega_{4 i}$ & $\mathrm{~A}_{1}+\mathrm{B}_{2}$ \\
\hline 1005 & $\mathrm{w}$ & $\omega_{6}+\omega_{947}$ & $\left(A_{2}\right)+A_{1}+B_{2}$ \\
\hline 1067 & $\mathrm{w}$ & $\omega_{8}+\omega_{3}$ & $\mathrm{~B}_{1}$ \\
\hline 1112 & $\mathrm{w}$ & $\omega_{1}+\omega_{3}$ & $\mathbf{A}_{1}$ \\
\hline 1144 & w & $\omega_{6}+\omega_{3}$ & $\mathrm{~B}_{2}$ \\
\hline 1171 & $\mathrm{vw}$ & $\omega_{6}+\omega_{9}+\omega_{5}$ & $A_{1}$ \\
\hline 1190 & $\nabla W$ & $\omega_{1}+2 \omega_{9}$ & $\mathrm{~A}_{1}$ \\
\hline 1221 & $\mathrm{vW}$ & $\omega_{8}+\omega_{3}+\omega_{5}$ & $\mathrm{~B}_{2}$ \\
\hline 1241 & $\nabla w$ & $\omega_{1}+\omega_{3}+\omega_{2}$ & $\mathrm{~A}_{1}$ \\
\hline 1256 & vw & $\omega_{6}+\omega_{3}+\omega_{2}$ & $\mathrm{~B}_{2}$ \\
\hline 1307 & w & $\omega_{8}+\omega_{3}+\omega_{9}$ & $A_{1}$ \\
\hline 1362 & w & $2 \omega_{8}$ & $A_{1}$ \\
\hline 1416 & $\mathrm{w}$ & $\omega_{1}+\omega_{8}$ & $\mathrm{~B}_{1}$ \\
\hline 1470 & $v w$ & $2 \omega_{1}$ & $A_{1}$ \\
\hline 1503 & $\mathrm{~m}$ & $\omega_{1}+\omega_{6}$ & $\mathrm{~B}_{2}$ \\
\hline 1540 & $\nabla w$ & $2 \omega_{6}$ & $\mathbf{A}_{1}$ \\
\hline 1592 & $\mathrm{w}$ & $2 \omega_{8}+\omega_{9}$ & $\mathrm{~B}_{1}$ \\
\hline
\end{tabular}

TABLE 4. The observed bands and their assignments of $\mathrm{CHF}_{3}$, $\mathrm{CHF}_{2} \mathrm{Cl}, \mathrm{CHFCl}_{2}$, and $\mathrm{CHCl}_{3}$

\begin{tabular}{|c|c|c|c|}
\hline$\nu$ & $\begin{array}{l}\text { Inten- } \\
\text { sity }\end{array}$ & Band & Symmetry \\
\hline \multicolumn{4}{|c|}{ Molecule $\mathrm{CHF}_{3}$ (Freon 23) molecular symmetry $\mathrm{C}_{3 \mathrm{v}}$} \\
\hline \multicolumn{4}{|l|}{$\mathrm{cm}^{-1}$} \\
\hline 507 & $\mathrm{~m}$ & $\omega_{25}$ & $\mathbf{E}$ \\
\hline 700 & $\mathrm{~m}$ & $\omega_{4}$ & $A_{1}$ \\
\hline 870 & $\mathrm{w}$ & $\omega_{68}-\omega_{25}$ & $\mathrm{~A}_{1}$ \\
\hline 1014 & w & $2 \omega_{25}$ & \\
\hline 1035 & VW & $2 \omega_{25}$ & $\mathbf{L}+\mathbf{A}_{1}$ \\
\hline $1150 ?$ & vs & $\omega_{3}$ & $A_{1}$ \\
\hline 1152 & vs & $\omega_{79}$ & $\mathbf{E}$ \\
\hline 1206 & $\mathrm{~s}$ & $\omega_{25}+\omega_{4}$ & $\mathbf{E}$ \\
\hline 1372 & $\mathrm{~m}$ & $\omega_{68}$ & E \\
\hline 1647 & $\mathrm{~m}$ & $\omega_{25}+\omega_{79}$ & $2 \mathrm{E}+\mathbf{A}_{1}\left(+\mathbf{A}_{2}\right)$ \\
\hline
\end{tabular}

TABLE 4. The observed bands and their assignments of $\mathrm{CHF}_{3}$, $\mathrm{CHF}_{2} \mathrm{Cl}, \mathrm{CHFCl}_{2}$, and $\mathrm{CHCl}_{2}$-Continued

\begin{tabular}{|c|c|c|c|}
\hline$\nu$ & $\begin{array}{l}\text { Inten- } \\
\text { sity }\end{array}$ & Band & Symmetry \\
\hline \multicolumn{4}{|c|}{ Molecule $\mathrm{CHF}_{3}$ (Freon 23) molecular symmetry $\mathrm{C}_{3}$} \\
\hline \multicolumn{4}{|l|}{$\mathrm{cm}^{-1}$} \\
\hline 1842 & $\mathrm{~m}$ & $\begin{array}{c}\left(\omega_{25}+\omega_{68} ?\right) \\
\omega_{4}+\omega_{3} \\
\omega_{4}+\omega_{79}\end{array}$ & $\begin{array}{c}\mathrm{E}+\mathrm{A}_{1}\left(+\mathrm{A}_{2}\right) \\
\mathrm{A}_{1} \\
\mathrm{E}\end{array}$ \\
\hline 2045 & vw & $\omega_{4}+\omega_{68}$ & E \\
\hline 2278 & $\mathrm{~m}$ & $\begin{array}{c}\omega_{3}+\omega_{79} \\
2 \omega_{79}\end{array}$ & $\begin{array}{l}2 \mathrm{E} \\
\mathrm{A}_{1}\end{array}$ \\
\hline 2523 & $\mathrm{~m}$ & $\begin{array}{c}\omega_{3}+\omega_{68} \\
\omega_{79}+\omega_{69}\end{array}$ & $\begin{array}{c}2 \mathrm{E} \\
\mathrm{A}_{1}\left(+\mathrm{A}_{2}\right)\end{array}$ \\
\hline 2716 & $\mathrm{w}$ & $2 \omega_{68}$ & $\mathrm{E}+\mathrm{A}_{1}$ \\
\hline 3031 & $\mathrm{~s}$ & $\omega_{1}$ & $A_{1}$ \\
\hline 3407 & $\mathrm{vw}$ & $2 \omega_{68}+\omega_{4}$ & $\mathrm{E}+\mathrm{A}_{1}$ \\
\hline 3445 & $\mathrm{vw}$ & $3 \omega 79$ & $\mathbf{L}+\mathrm{A}_{1}$ \\
\hline 4212 & $\mathrm{w}$ & $\begin{array}{l}\omega_{3}+\omega_{1} \\
\omega_{79}+\omega_{1}\end{array}$ & $\begin{array}{l}\mathrm{A}_{1} \\
\mathrm{E}\end{array}$ \\
\hline 4448 & $\mathrm{vw}$ & $\omega_{68}+\omega_{1}$ & E \\
\hline \multicolumn{4}{|c|}{ Molecule $\mathrm{CHF}_{2} \mathrm{Cl}$ (Freon 22) molecular symmetry $\mathrm{C}_{8}$} \\
\hline 365 & w & $\omega_{5}$ & $\mathrm{~A}^{\prime \prime}$ \\
\hline 422 & $\mathrm{w}$ & $\omega_{2}$ & $\mathrm{~A}^{\prime}$ \\
\hline 595 & $\mathrm{~s}$ & $\omega_{4}$ & $A^{\prime}$ \\
\hline 809 & vs & $\omega_{7}$ & $\mathrm{~A}^{\prime}$ \\
\hline 833 & $\mathrm{~m}$ & $2 \omega_{2}$ & $\mathrm{~A}^{\prime}$ \\
\hline 1116 & vs & $\omega 9$ & $\mathrm{~A}^{\prime \prime}$ \\
\hline 1178 & $\mathrm{~m}$ & $\begin{array}{c}\omega_{3} \\
\omega_{5}+\omega_{7}\end{array}$ & $\begin{array}{l}\mathrm{A}^{\prime} \\
\mathrm{A}^{\prime}\end{array}$ \\
\hline 1198 & $\mathrm{w}$ & $2 \omega_{4}$ & $A^{\prime}$ \\
\hline 1244 & vw & $\omega_{2}+\omega_{7}$ & $A^{\prime}$ \\
\hline 1311 & $\mathrm{~s}$ & $\omega_{6}$ & $A^{\prime}$ \\
\hline 1347 & $\mathrm{~m}$ & $\omega_{8}$ & $A^{\prime \prime}$ \\
\hline $1625 ?$ & $\mathrm{~W}$ & $\begin{array}{c}2 \omega_{7} \\
\omega_{5}+\omega_{6}\end{array}$ & $\begin{array}{l}\mathrm{A}^{\prime} \\
\mathrm{A}^{\prime \prime}\end{array}$ \\
\hline $1695 ?$ & $\nabla w$ & $\begin{array}{l}\omega_{5}+\omega_{8} \\
\omega_{4}+\omega_{9}\end{array}$ & $\begin{array}{l}\mathrm{A}^{\prime} \\
\mathrm{A}^{\prime}\end{array}$ \\
\hline 2222 & $\mathrm{~m}$ & $2 \omega_{9}$ & $\mathrm{~A}^{\prime}$ \\
\hline 3023 & $\mathrm{~m}$ & $\omega_{1}$ & $A^{\prime}$ \\
\hline $3769 ?$ & VW & $\omega_{7}+\omega_{1} ?$ & $\mathrm{~A}^{\prime}$ \\
\hline 4167 & $\mathrm{vW}$ & $\omega_{9}+\omega_{1}$ & $\mathrm{~A}^{\prime \prime}$ \\
\hline \multicolumn{4}{|c|}{ 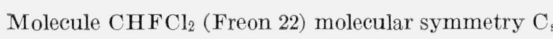 } \\
\hline$[276](\mathrm{R})$ & -..- & $\omega_{2}$ & $\mathrm{~A}^{\prime}$ \\
\hline 368 & w & $\omega_{5}$ & $\mathrm{~A}^{\prime \prime}$ \\
\hline 458 & $\mathrm{w}$ & $\begin{array}{c}\omega_{4} \\
\omega_{2}+\omega_{5} ?\end{array}$ & $\begin{array}{l}\mathbf{A}^{\prime} \\
\mathbf{A}^{\prime \prime}\end{array}$ \\
\hline 742 & $\mathrm{~s}$ & $\begin{array}{c}\left(\omega_{2}+\omega_{4}\right) \\
\omega_{3} ?\end{array}$ & $\mathbf{A}^{\prime}$ \\
\hline 804 & vs & $\omega_{9}$ & $\mathrm{~A}^{\prime \prime}$ \\
\hline 835 & $\mathrm{~s}$ & $\omega_{5}+\omega_{4}$ & $\mathrm{~A}^{\prime \prime}$ \\
\hline $955 ?$ & $\mathrm{w}$ & $\begin{array}{l}\omega_{6}-\omega_{5} \\
\omega_{8}-\omega_{2}\end{array}$ & $\begin{array}{l}\mathrm{A}^{\prime \prime} \\
\mathrm{A}^{\prime \prime}\end{array}$ \\
\hline 1079 & $\mathrm{~s}$ & $\begin{array}{c}\omega_{2}+\omega_{9} \\
\omega_{7}\end{array}$ & $\begin{array}{l}\mathbf{A}^{\prime \prime} \\
\mathbf{A}^{\prime}\end{array}$ \\
\hline 1166 & $\mathrm{w}$ & $\omega_{5}+\omega_{9}$ & $A^{\prime}$ \\
\hline 1242 & $\mathrm{~s}$ & $\omega_{8}$ & $\mathrm{~A}^{\prime \prime}$ \\
\hline 1260 & $\mathrm{w}$ & $\omega_{4}+\omega_{9}$ & $\mathrm{~A}^{\prime \prime}$ \\
\hline 1304 & $\mathrm{~s}$ & $\omega_{\theta}$ & $\mathrm{A}^{\prime}$ \\
\hline 1494 & $w$ & $\omega_{5}+\omega_{7}$ & $\mathrm{~A}^{\prime \prime}$ \\
\hline 1536 & $\mathrm{~m}$ & $\omega_{4}+\omega_{7}$ & $\mathrm{~A}^{\prime}$ \\
\hline 1608 & $\mathrm{w}$ & $\begin{array}{c}2 \omega_{9} \\
\omega_{5}+\omega_{8}\end{array}$ & $\begin{array}{l}\mathrm{A}^{\prime} \\
\mathrm{A}^{\prime}\end{array}$ \\
\hline 2123 & $\mathrm{~m}$ & $\begin{array}{c}2 \omega_{7} \\
\omega_{9}+\omega_{6}\end{array}$ & $\begin{array}{l}\mathbf{A}^{\prime} \\
\mathbf{A}^{\prime \prime}\end{array}$ \\
\hline $\begin{array}{l}2280 ? \\
2463\end{array}$ & $\begin{array}{l}\mathrm{w} \\
\mathrm{w}\end{array}$ & $\begin{array}{c}\omega_{8}+\omega_{7} \\
2 \omega_{8}\end{array}$ & $\begin{array}{l}\mathrm{A}^{\prime \prime} \\
\mathrm{A}^{\prime}\end{array}$ \\
\hline
\end{tabular}


TABLE 4. The observed bands and their assigments of $\mathrm{CHF}_{3}$, $\mathrm{CHF}_{2} \mathrm{Cl}, \mathrm{CHFCl}_{2}$, and $\mathrm{CHCl}_{3}$-Continued

\begin{tabular}{|c|c|c|c|}
\hline$\nu$ & $\begin{array}{l}\text { Inten- } \\
\text { sity }\end{array}$ & Band & Symmetry \\
\hline \multicolumn{4}{|c|}{ 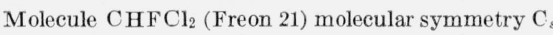 } \\
\hline \multicolumn{4}{|l|}{$\mathrm{cm}^{-1}$} \\
\hline 2557 & $\nabla \mathbf{W}$ & $\omega_{6}+\omega_{8}$ & $\mathrm{~A}^{\prime \prime}$ \\
\hline 2616 & $\nabla w$ & $2 \omega_{6}$ & $A^{\prime}$ \\
\hline 3023 & $\mathrm{~m}$ & $\omega_{1}$ & $A^{\prime}$ \\
\hline 3184 & vw & $3 \omega_{7}$ & $\mathrm{~A}^{\prime}$ \\
\hline $3780 ?$ & vw & $\omega_{1}+\omega_{3}$ & $\mathrm{~A}^{\prime}$ \\
\hline 4144 & vw & $\omega_{7}+\omega_{1}$ & $A^{\prime}$ \\
\hline 4303 & w & $\omega_{6}+\omega_{1}$ & $A^{\prime}$ \\
\hline 4398 & $\nabla w$ & $4 \omega_{7} ?$ & $A^{\prime}$ \\
\hline \multicolumn{4}{|c|}{ Molecule $\mathrm{CHCl}_{3}$ molecular symmetry $\mathrm{C}_{3 \mathrm{v}}$} \\
\hline$[261](\mathrm{R})$ & $\ldots$ & $\omega_{25}$ & $\mathrm{E}$ \\
\hline 370 & $\mathrm{~m}$ & $\omega_{4}$ & $\mathbf{A}_{1}$ \\
\hline 392 & w & $\omega_{3}-\omega_{25}$ & $\mathrm{E}+\mathrm{A}_{1}$ \\
\hline 488 & $\mathrm{w}$ & $2 \omega_{25}$ & $\mathrm{E}+\mathrm{A}_{1}$ \\
\hline & & $\omega_{79}-\omega_{25}$ & $\mathrm{~A}_{1}$ \\
\hline 629 & $\mathrm{~m}$ & $\omega_{4}+\omega_{25}$ & $\mathrm{E}$ \\
\hline 667 & $\mathbf{s}$ & $\omega_{3}$ & $A_{1}$ \\
\hline 755 & ys & $\omega_{79}$ & $\mathrm{E}$ \\
\hline & & $2 \omega_{4}$ & $A_{1}$ \\
\hline 849 & $\mathrm{~m}$ & $\omega_{68}-\omega_{4}$ & $\mathrm{E}$ \\
\hline 929 & $\mathrm{~m}$ & $\omega_{3}+\omega_{25}$ & $\mathrm{E}$ \\
\hline 951 & w & $\omega_{68}-\omega_{25}$ & $A_{1}$ \\
\hline 1031 & $\mathrm{~m}$ & $\omega_{3}+\omega_{4}$ & $\mathrm{~A}_{1}$ \\
\hline 1049 & $\mathrm{~m}$ & $\omega_{79}+\omega_{25}$ & $\mathrm{E}+2 \mathrm{~A}_{1}$ \\
\hline 1145 & $\mathrm{w}$ & $\omega_{79}+\omega_{4}$ & $\mathrm{E}$ \\
\hline 1214 & $\mathrm{~s}$ & $\omega_{68}$ & $\mathrm{E}$ \\
\hline 1333 & w & $2 \omega_{3}$ & $\mathbf{A}_{1}$ \\
\hline 1393 & $\mathrm{~m}$ & $\omega_{79}+\omega_{4}+\omega_{25}$ & $\mathbf{E}+2 \mathbf{A}_{1}$ \\
\hline 1420 & $\mathrm{~m}$ & $\omega_{3}+\omega_{79}$ & E \\
\hline 1524 & $\mathrm{~m}$ & $2 \omega 79$ & $\mathrm{E}+\mathrm{A}_{1}$ \\
\hline 2410 & $\mathrm{~m}$ & $2 \omega_{68}$ & $\mathbf{E}+\mathbf{A}_{1}$ \\
\hline 3040 & $\mathrm{~m}$ & $\omega_{1}$ & $\mathbf{A}_{1}$ \\
\hline 3597 & w & $3 \omega_{68}$ & $\mathrm{E}+\mathrm{A}_{1}+\mathrm{A}_{2}$ \\
\hline 3663 & w & $\omega_{1}+\omega_{3}$ & $\mathrm{~A}_{1}$ \\
\hline 4202 & $\mathrm{~m}$ & $\omega_{1}+\omega_{68}$ & $\mathrm{E}$ \\
\hline
\end{tabular}

TABLE 5. The observed bands and their assignments of $\mathrm{CHCl}_{2} \mathrm{Br}, \mathrm{CHClBr}_{2}, \mathrm{CHBr}_{3}$, and $\mathrm{CHI}_{3}$

\begin{tabular}{|c|c|c|c|}
\hline$\nu$ & $\begin{array}{l}\text { Inten- } \\
\text { sity }\end{array}$ & Band & Symmetry \\
\hline \multicolumn{4}{|c|}{$\mathrm{Molecule}_{\mathrm{CHCl}} \mathrm{Br}_{2}$ molecular symmetry $\mathrm{C}_{s}$} \\
\hline \multicolumn{4}{|l|}{$\mathrm{cm}^{-1}$} \\
\hline$\lceil 215\rceil(\mathrm{R})$ & -... & $\omega_{5}$ & $\mathrm{~A}^{\prime \prime}$ \\
\hline $220(\mathrm{R})$ & .... & $\omega_{2}$ & $A^{\prime}$ \\
\hline 330 & $\mathrm{~m}$ & $\omega 4$ & $\mathrm{~A}^{\prime}$ \\
\hline 383 & w & $\omega_{3}-\omega_{25}$ & $\mathrm{~A}^{\prime}+\mathrm{A}^{\prime \prime}$ \\
\hline 437 & w & $2 \omega_{25}$ & $2 \mathrm{~A}^{\prime}+\mathrm{A}^{\prime \prime}$ \\
\hline 495 & w & $\omega_{7}-\omega_{25}$ & $\mathbf{A}^{\prime}+\mathbf{A}^{\prime \prime}$ \\
\hline 552 & w & $\begin{array}{l}\omega_{4}+\omega_{25} \\
\omega_{9}-\omega_{25}\end{array}$ & $\begin{array}{l}\mathrm{A}^{\prime}+\mathrm{A}^{\prime \prime} \\
\mathrm{A}^{\prime}+\mathrm{A}^{\prime \prime}\end{array}$ \\
\hline 604 & $\mathrm{~s}$ & $\omega_{3}$ & $\mathrm{~A}^{\prime}$ \\
\hline 643 & $m$ & $2 \omega 4$ & $\mathrm{~A}^{\prime}$ \\
\hline 719 & vs & $\omega_{7}$ & $A^{\prime}$ \\
\hline 760 & vs & $\omega_{9}$ & $A^{\prime \prime}$ \\
\hline 795 & $\mathrm{~m}$ & $\omega_{3}+\omega_{5}$ & $\mathrm{~A}^{\prime \prime}$ \\
\hline 814 & $\mathbf{w}$ & $\omega_{3}+\omega_{2}$ & $\mathrm{~A}^{\prime}$ \\
\hline 843 & $v w$ & . & - \\
\hline 858 & $w$ & $\omega_{6}-\omega_{4}$ & $\mathbf{A}^{\prime \prime}$ \\
\hline 884 & $\mathbf{w}$ & $\omega_{8}-\omega_{4}$ & $\mathrm{~A}^{\prime}$ \\
\hline
\end{tabular}

TABLE 5. The observed bands and their assignments of $\mathrm{CHCl}_{2} \mathrm{Br}, \mathrm{CHClBr}_{2}, \mathrm{CHBr}_{3}$, and $\mathrm{CHI}_{3}$ - Continued

\begin{tabular}{|c|c|c|c|}
\hline$\nu$ & $\begin{array}{l}\text { Inten- } \\
\text { sity }\end{array}$ & Band & Symmetry \\
\hline \multicolumn{4}{|c|}{ Molecule $\mathrm{CHCl}_{2} \mathrm{Br}$ molecular symmetry $\mathrm{C}_{8}$} \\
\hline \multicolumn{4}{|l|}{$\mathrm{cm}^{-1}$} \\
\hline 916 & $\mathrm{w}$ & $\omega_{7}+\omega_{25}$ & $\mathrm{~A}^{\prime}+\mathrm{A}^{\prime \prime}$ \\
\hline 924 & $\mathrm{w}$ & $\omega_{3}+\omega_{4}$ & $\mathrm{~A}^{\prime}$ \\
\hline 942 & $\mathrm{w}$ & $\begin{array}{l}\omega_{9}+\omega_{25} \\
\omega_{6}-\omega_{25}\end{array}$ & $\begin{array}{l}\mathrm{A}^{\prime}+\mathrm{A}^{\prime \prime} \\
\mathrm{A}^{\prime}+\mathrm{A}^{\prime \prime}\end{array}$ \\
\hline 1004 & VW & $\omega_{8}-\omega_{25}$ & $\mathrm{~A}^{\prime}+\mathrm{A}^{\prime \prime}$ \\
\hline 1052 & $\mathrm{w}$ & - n. & - \\
\hline 1066 & $\mathrm{w}$ & $\omega_{7}+\omega_{4}$ & $\mathrm{~A}^{\prime}$ \\
\hline 1114 & $\mathrm{w}$ & $\omega_{9}+\omega_{4}$ & $\mathrm{~A}^{\prime \prime}$ \\
\hline 1168 & $\mathrm{~s}$ & $\omega_{6}$ & $\mathrm{~A}^{\prime}$ \\
\hline 1189 & $\mathrm{~m}$ & $2 \omega_{3}$ & $\mathrm{~A}^{\prime}$ \\
\hline 1211 & $\mathrm{~s}$ & $\omega_{8}$ & $A^{\prime \prime}$ \\
\hline 1312 & $\mathrm{w}$ & $\omega_{3}+\omega_{7}$ & $A^{\prime}$ \\
\hline 1385 & vw & $\left\{\begin{array}{l}\omega_{3}+\omega_{9} \\
\omega_{6}+\omega_{25}\end{array}\right.$ & $\begin{array}{c}\mathrm{A}^{\prime \prime} \\
\mathrm{A}^{\prime}+\mathrm{A}^{\prime \prime}\end{array}$ \\
\hline 1414 & $\mathrm{vw}$ & $\begin{array}{c}2 \omega_{7} \\
\omega_{8}+\omega_{25}\end{array}$ & $\begin{array}{c}\mathrm{A}^{\prime} \\
\mathrm{A}^{\prime}+\mathrm{A}^{\prime \prime}\end{array}$ \\
\hline 1506 & $\mathrm{w}$ & $2 \omega_{9}$ & $\mathrm{~A}^{\prime}$ \\
\hline 1592 & w & $\omega_{68}+\omega_{4}$ & $\mathrm{~A}^{\prime}+\mathrm{A}^{\prime \prime}$ \\
\hline 2421 & $\mathrm{w}$ & $2 \omega_{68}$ & $2 \mathrm{~A}^{\prime}+\mathrm{A}^{\prime \prime}$ \\
\hline 3040 & $\mathrm{~s}$ & $\omega_{1}$ & $\mathbf{A}^{\prime}$ \\
\hline 3077 & $\mathrm{vw}$ & $2 \omega_{6}+\omega_{7}$ & $\mathrm{~A}^{\prime}$ \\
\hline 3802 & $\mathrm{vw}$ & $\omega_{1}+\omega_{9}$ & $A^{\prime \prime}$ \\
\hline 4237 & $\mathrm{~m}$ & $\omega_{1}+\omega_{6}$ & $\mathrm{~A}^{\prime}$ \\
\hline 4291 & w & $\omega_{1}+\omega_{8}$ & $A^{\prime \prime}$ \\
\hline 4504 & $\mathrm{vW}$ & $\omega_{1}+2 \omega_{9}$ & $\mathrm{~A}^{\prime}$ \\
\hline \multicolumn{4}{|c|}{ Molecule $\mathrm{CHClBr}_{2}$ molecular symmetry $\mathrm{C}_{\mathrm{s}}$} \\
\hline [168] (R) & -...- & $\omega_{2}$ & $\mathrm{~A}^{\prime}$ \\
\hline$[201](\mathrm{R})$ & 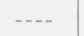 & $\omega_{5}$ & $A^{\prime \prime}$ \\
\hline 278 & $\mathrm{w}$ & $\omega_{4}$ & $\mathrm{~A}^{\prime}$ \\
\hline 383 & w & $\begin{array}{c}\omega_{3}-\omega_{25} \\
2 \omega_{25}\end{array}$ & $\begin{array}{c}A^{\prime}+A^{\prime \prime} \\
2 A^{\prime}+A^{\prime \prime}\end{array}$ \\
\hline & & $\omega_{4}+\omega_{2}$ & $\mathrm{~A}^{\prime}$ \\
\hline 460 & w & $\omega_{9}-\omega_{5}$ & $\mathrm{~A}^{\prime}$ \\
\hline 492 & $\mathrm{w}$ & $\omega_{4}+\omega_{5}$ & $A^{\prime \prime}$ \\
\hline 570 & $\mathrm{~s}$ & $\begin{array}{c}\omega_{9}-\omega_{2} \\
\omega_{3}\end{array}$ & $\begin{array}{l}\mathrm{A}^{\prime \prime} \\
\mathrm{A}^{\prime}\end{array}$ \\
\hline 657 & vs & $\omega_{9}$ & $\mathrm{~A}^{\prime \prime}$ \\
\hline 733 & $\mathrm{~m}$ & $\omega_{3}+\omega_{2}$ & $\mathrm{~A}^{\prime}$ \\
\hline 746 & $\mathrm{~s}$ & $\omega_{7}$ & $\mathbf{A}^{\prime}$ \\
\hline 796 & $\mathrm{~m}$ & $\omega_{3}+\omega_{5}$ & $\mathbf{A}^{\prime \prime}$ \\
\hline 821 & $\mathrm{w}$ & $\omega_{2}+\omega_{9}$ & $\mathbf{A}^{\prime \prime}$ \\
\hline 844 & w & $\begin{array}{l}\omega_{3}+\omega_{4} \\
\omega_{9}+\omega_{5}\end{array}$ & $\begin{array}{l}\mathrm{A}^{\prime} \\
\mathrm{A}^{\prime}\end{array}$ \\
\hline 937 & w & $\left\{\begin{array}{c}\omega_{7}+\omega_{25} \\
\omega_{9}+\omega_{4}\end{array}\right.$ & $\begin{array}{c}\mathrm{A}^{\prime}+\mathrm{A}^{\prime \prime} \\
\mathrm{A}^{\prime \prime}\end{array}$ \\
\hline 1004 & w & $\omega_{7}+\omega_{4}$ & $\mathbf{A}^{\prime}$ \\
\hline 1029 & $\nabla w$ & $\omega_{6}-\omega_{2}$ & $\mathrm{~A}^{\prime}$ \\
\hline 1053 & w & $\omega_{2}+\omega_{5}+\omega_{9}$ & $\mathrm{~A}^{\prime}+\mathrm{A}^{\prime \prime}$ \\
\hline 1144 & vS & $\left\{\begin{array}{c}\omega_{8} \\
2 \omega_{3}\end{array}\right.$ & $\begin{array}{l}\mathbf{A}^{\prime \prime} \\
\mathbf{A}^{\prime}\end{array}$ \\
\hline 1189 & S & $\omega_{6}$ & $\mathbf{A}^{\prime}$ \\
\hline 1218 & $\mathrm{w}$ & $\omega_{3}+\omega_{9}$ & $\mathbf{A}^{\prime \prime}$ \\
\hline 1309 & $\mathrm{w}$ & $2 \omega_{9}$ & $\mathbf{A}^{\prime}$ \\
\hline 1340 & $\mathrm{VW}$ & $\omega_{8}+\omega_{5}$ & $\mathbf{A}^{\prime \prime}$ \\
\hline 1340 & Vw & $\omega_{6}+\omega_{2}$ & $\mathbf{A}^{\prime \prime}$ \\
\hline 1410 & $\mathrm{w}$ & $\omega_{7}+\omega_{9}$ & $\mathbf{A}^{\prime \prime}$ \\
\hline 1473 & $\mathrm{w}$ & $\omega_{6}+\omega_{4}$ & $\mathbf{A}^{\prime}$ \\
\hline 1488 & $\mathrm{w}$ & $2 \omega 7$ & $\mathbf{A}^{\prime}$ \\
\hline $1592 ?$ & $\mathrm{w}$ & $\omega_{5}+\omega_{7}+\omega_{9}$ & $\mathbf{A}^{\prime \prime}$ \\
\hline 2278 & $\mathrm{~m}$ & $2 \omega_{68}$ & $2 \mathrm{~A}^{\prime}+\mathrm{A}^{\prime \prime}$ \\
\hline 3034 & $\mathrm{~s}$ & $\omega_{1}$ & $\mathbf{A}^{\prime}$ \\
\hline 4203 & $\mathrm{~m}$ & $\omega_{1}+\omega_{8}$ & $\mathbf{A}^{\prime \prime}$ \\
\hline 4257 & $\mathrm{w}$ & $\omega_{1}+\omega_{6}$ & $\mathbf{A}^{\prime}$ \\
\hline
\end{tabular}


TABLE 5. The observed bands and their assignments of $\mathrm{CHCl}_{2} \mathrm{Br}, \mathrm{CHClBr}_{2}, \mathrm{CHBr}_{3}$, and $\mathrm{CHI}_{3}$ - Continued

\begin{tabular}{|c|c|c|c|}
\hline$\nu$ & $\begin{array}{l}\text { Inten- } \\
\text { sity }\end{array}$ & Band & Symmetry \\
\hline \multicolumn{4}{|c|}{ Molecule $\mathrm{CHBr}_{3}$ molecular symmetry $\mathrm{C}_{3 \mathrm{v}}$} \\
\hline $\mathrm{cm}^{-1}$ & & & \\
\hline$[154](\mathrm{R})$ & -...- & $\omega_{25}$ & $\mathrm{E}$ \\
\hline$[222](\mathrm{R})$ & -..- & $\omega_{4}$ & $\mathbf{A}_{1}$ \\
\hline$[300 ?](\mathrm{R})$ & -... & $2 \omega_{25}$ & $\mathrm{E}+\mathrm{A}_{1}$ \\
\hline 380 & $\mathrm{~m}$ & $\omega_{4}+\omega_{25}$ & E \\
\hline 389 & $\mathrm{~m}$ & $\omega_{3}-\omega_{25}$ & $\mathbf{E}$ \\
\hline 435 & w & $2 \omega_{4}$ & $\mathbf{A}_{1}$ \\
\hline 501 & $\mathrm{w}$ & $\omega_{79}-\omega_{25}$ & $\mathbf{A}_{1}$ \\
\hline 541 & $\mathrm{~m}$ & $\omega_{3}$ & $\mathbf{A}_{1}$ \\
\hline 668 & vs & $\omega_{79}$ & $\mathbf{E}$ \\
\hline $694 ?$ & $\mathrm{~m}$ & $\omega_{3}+\omega_{25}$ & $\mathrm{E}$ \\
\hline 762 & w & $\omega_{3}+\omega_{4}$ & $\mathbf{A}_{1}$ \\
\hline 873 & $\mathrm{w}$ & $\omega_{79}+\omega_{4}$ & $\mathrm{E}$ \\
\hline 1078 & w & $2 \omega_{3}$ & $\mathrm{~A}_{1}$ \\
\hline 1142 & vs & $\omega_{68}$ & $\mathrm{E}$ \\
\hline 1188 & $\mathrm{~m}$ & $\omega_{3}+\omega_{79}$ & $\mathrm{E}$ \\
\hline 1295 & $\mathrm{~m}$ & $\begin{array}{c}2 \omega_{79} \\
\omega_{68}+\omega_{25}\end{array}$ & $\begin{array}{l}\mathrm{E}+\mathrm{A}_{1} \\
\mathrm{E}+2 \mathrm{~A}_{1}\end{array}$ \\
\hline $1493 ?$ & vw & $2 \omega_{79}+\omega_{25}$ & $2 \mathrm{E}+\mathrm{A}_{1}$ \\
\hline $1597 ?$ & $\mathrm{vw}$ & $\omega_{3}+\omega_{68}$ & E \\
\hline 2257 & w & $2 \omega_{68}$ & $\mathbf{E}+\mathbf{A}_{1}$ \\
\hline 3040 & $\mathrm{~m}$ & $\omega_{1}$ & $\mathrm{~A}_{1}$ \\
\hline 4202 & w & $\omega_{1}+\omega_{68}$ & $\mathrm{E}$ \\
\hline \multicolumn{4}{|c|}{ Molecule $\mathrm{CHBr}_{3}$ molecular symmetry $\mathrm{C}_{3 \mathrm{v}}$} \\
\hline$[92] ?(\mathrm{R})$ & .... & $\omega_{25}$ & $\mathrm{E}$ \\
\hline$[145] ?(\mathrm{R})$ & $\ldots$ & $\omega_{4}$ & $\mathrm{~A}_{1}$ \\
\hline$[385](\mathrm{R})$ & $-\ldots$ & $\omega 3$ & $\mathrm{~A}_{1}$ \\
\hline 581 & $\mathrm{~m}$ & $\omega_{79}$ & $\mathrm{E}$ \\
\hline 1064 & $\mathrm{~m}$ & $\omega_{68}$ & $\mathrm{E}$ \\
\hline$[3040](\mathrm{R})$ & $\ldots$ & $\omega_{1}$ & $A_{1}$ \\
\hline
\end{tabular}

TABLE 6. The observed bands and their assignments of $\mathrm{CH}_{2} \mathrm{~F}_{2}$, $\mathrm{CH}_{2} \mathrm{Cl}_{2}$, and $\mathrm{CH}_{2} \mathrm{I}_{2}$

\begin{tabular}{|c|c|c|c|}
\hline$\nu$ & $\begin{array}{l}\text { Inten- } \\
\text { sity }\end{array}$ & Band & Symmetry \\
\hline \multicolumn{4}{|c|}{$\begin{array}{c}\text { Molecule } \mathrm{CH}_{2} \mathrm{~F}_{2} \text { (Freon } 32 \text { ) molecular } \\
\text { symmetry } \mathrm{C}_{2 \mathrm{v}}\end{array}$} \\
\hline $\mathrm{cm}^{-1}$ & & & \\
\hline 529 & $\mathrm{~s}$ & $\omega 4$ & $\mathbf{A}_{1}$ \\
\hline 757 & w & $\omega_{5}-\omega_{4}$ & $\mathrm{~A}_{2}$ \\
\hline 816 & $\mathrm{w}$ & - & - \\
\hline 910 & w & $\omega_{8}-\omega_{4}$ & $\mathrm{~B}_{1}$ \\
\hline 1090 & vs & $\omega_{9}$ & $\mathrm{~B}_{1}$ \\
\hline 1116 & $\mathrm{~s}$ & $\omega_{2}$ & $\mathbf{A}_{1}$ \\
\hline 1176 & $\mathrm{~m}$ & $\omega_{7}$ & $\mathrm{~B}_{2}$ \\
\hline 1262 & $\mathrm{w}$ & $\omega_{5}$ & $\mathbf{A}_{2}$ \\
\hline 1349 & $\mathrm{w}$ & - & -...- \\
\hline 1435 & $\mathrm{~s}$ & $\omega_{8}$ & $\mathrm{~B}_{1}$ \\
\hline (1508) & $-\cdots$ & $\omega_{3}$ & $\mathbf{A}_{1}$ \\
\hline 1623 & $\mathrm{vw}$ & $\omega_{4}+\omega_{29}$ & $\mathrm{~A}_{1}+\mathrm{B}_{1}$ \\
\hline 2183 & $\mathrm{~m}$ & $2 \omega_{29}$ & $2 \mathrm{~A}_{1}+\mathrm{B}_{1}$ \\
\hline 2551 & VW & $\begin{array}{c}2 \omega_{5} \\
\omega_{8}+\omega_{29}\end{array}$ & $\begin{array}{c}\mathrm{A}_{1} \\
\mathrm{~A}_{1}+\mathrm{B}_{1}\end{array}$ \\
\hline 2838 & $\mathrm{~m}$ & $2 \omega_{8}$ & $\mathrm{~A}_{1}$ \\
\hline 2945 & $\mathrm{~m}$ & $\omega_{3}+\omega_{8}$ & $\mathrm{~B}_{1}$ \\
\hline 2949 & $\mathrm{~s}$ & $\omega_{1}$ & $A_{1}$ \\
\hline 3012 & $\mathrm{~s}$ & $\omega_{6}$ & $\mathrm{~B}_{2}$ \\
\hline 4161 & w & $\begin{array}{l}\omega_{1}+\omega_{7} \\
\omega_{6}+\omega_{29}\end{array}$ & $2 \mathrm{~B}_{2}+\mathrm{A}_{2}$ \\
\hline 4550 & w & $\omega_{6}+\omega_{3}$ & $\mathrm{~B}_{2}$ \\
\hline
\end{tabular}

TABLE 6. The observed bands and their assignments of $\mathrm{CH}_{2} \mathrm{~F}_{2}$, $\mathrm{CH}_{2} \mathrm{Cl}_{2}$, and $\mathrm{CH}_{2} \mathrm{I}_{2}$-Continued

\begin{tabular}{|c|c|c|c|}
\hline$\nu$ & $\begin{array}{l}\text { Inten- } \\
\text { sity }\end{array}$ & Band & Symmetry \\
\hline \multicolumn{4}{|c|}{ Molecule $\mathrm{CH}_{2} \mathrm{Cl}_{2}$ molecular symmetry $\mathrm{C}_{2 \mathrm{v}}$} \\
\hline \multicolumn{4}{|l|}{$\mathrm{cm}^{-}$} \\
\hline 286 & $\mathrm{w}$ & $\omega 4$ & $\mathbf{A}_{1}$ \\
\hline 444 & w & $\omega_{9}-\omega_{4}$ & $\mathrm{~B}_{1}$ \\
\hline 706 & $\mathrm{~m}$ & $\omega_{2}$ & $\mathbf{A}_{1}$ \\
\hline 742 & vs & $\omega_{9}$ & $\mathrm{~B}_{1}$ \\
\hline 898 & $\mathrm{~m}$ & $\omega 7$ & $\mathrm{~B}_{2}$ \\
\hline 991 & $\mathrm{w}$ & $\omega_{2}+\omega_{4}$ & $\mathbf{A}_{1}$ \\
\hline 1159 & vW & $\omega_{5}$ & $\mathrm{~A}_{2}$ \\
\hline 1262 & $\mathrm{~s}$ & $\omega_{8}$ & $\mathrm{~B}_{1}$ \\
\hline 1424 & $\mathrm{~m}$ & $\omega_{3}$ & $A_{1}$ \\
\hline 1562 & vw & $\omega_{4}+\omega_{8}$ & $\mathrm{~B}_{1}$ \\
\hline 1621 & VW & $\omega_{7}+\omega_{29}$ & $\mathbf{A}_{1}+\mathbf{B}_{2}$ \\
\hline 1945 & vw & $\omega_{2}+\omega_{8}$ & $\mathrm{~B}_{1}$ \\
\hline 2053 & vW & $\omega_{5}+\omega_{7}$ & $\mathrm{~B}_{1}$ \\
\hline 2132 & VW & $\omega_{3}+\omega_{2}$ & $\mathbf{A}_{1}$ \\
\hline 2155 & $\mathrm{vW}$ & $\omega_{3}+\omega_{9}$ & $\mathrm{~B}_{1}$ \\
\hline 2304 & $\mathrm{~m}$ & $\omega_{7}+\omega_{3}$ & $\mathrm{~B}_{2}$ \\
\hline 2410 & w & $\omega_{8}+\omega_{5}$ & $\mathrm{~B}_{2}$ \\
\hline 2525 & $\mathrm{w}$ & $2 \omega 8$ & $\mathbf{A}_{1}$ \\
\hline \multirow{2}{*}{2688} & \multirow{2}{*}{$\mathrm{m}$} & $\omega_{1}-\omega_{4}$ & $A_{1}$ \\
\hline & & $\omega_{3}+\omega_{8}$ & $\mathrm{~B}_{1}$ \\
\hline 2833 & $v w$ & $2 \omega_{3}$ & $\mathbf{A}_{1}$ \\
\hline 2985 & $\mathrm{~s}$ & $\omega_{1}$ & $\mathbf{A}_{1}$ \\
\hline 3048 & $\mathrm{~s}$ & $\omega_{6}$ & $\mathrm{~B}_{2}$ \\
\hline 3690 & w & $\omega_{1}+\omega_{2}$ & $A_{1}$ \\
\hline 3952 & $w$ & $\omega_{6}+\omega_{7}$ & $\mathrm{~A}_{1}$ \\
\hline 4219 & $\mathrm{~m}$ & $\omega_{6}+\omega_{5}$ & $\mathrm{~B}_{1}$ \\
\hline 4273 & $\mathrm{w}$ & $\omega_{6}+\omega_{8}$ & $\mathrm{~B}_{1}$ \\
\hline 4464 & $\mathrm{~m}$ & $\omega_{6}+\omega_{3}$ & $\mathrm{~B}_{2}$ \\
\hline \multicolumn{4}{|c|}{ Molecule $\mathrm{CH}_{2} \mathrm{I}_{2}$ molecular symmetry $\mathrm{C}_{2 \mathrm{v}}$} \\
\hline$[128](\mathrm{R})$ & $\ldots$ & $\omega_{4}$ & $\mathbf{A}_{1}$ \\
\hline 444 & W & $\omega_{9}-\omega_{4}$ & $\mathrm{~B}_{1}$ \\
\hline 486 & $\mathrm{~m}$ & $\omega_{2}$ & $A_{1}$ \\
\hline 572 & $\mathrm{~s}$ & $\omega_{9}$ & $\mathrm{~B}_{1}$ \\
\hline 639 & w & $\omega_{2}+\omega_{4}$ & $A_{1}$ \\
\hline 717 & $\mathrm{~m}$ & $\omega_{7}$ & $\mathrm{~B}_{2}$ \\
\hline 1035 & $W$ & $\omega_{5}$ & $\mathrm{~A}_{2}$ \\
\hline 1107 & vs & $\omega_{8}$ & $\mathrm{~B}_{1}$ \\
\hline 1182 & vw & $\omega_{2}+\omega_{7}$ & $\mathrm{~B}_{2}$ \\
\hline 1225 & $\mathrm{w}$ & $\omega_{4}+\omega_{8}$ & $\mathrm{~B}_{1}$ \\
\hline 1351 & $\mathrm{~m}$ & $\omega_{3}$ & $A_{1}$ \\
\hline 1435 & vw & $2 \omega_{7}$ & $\mathbf{A}_{1}$ \\
\hline 1597 & $\mathrm{w}$ & $\omega_{2}+\omega_{8}$ & $\mathrm{~B}_{1}$ \\
\hline 1748 & $\mathrm{w}$ & $\omega_{5}+\omega_{7}$ & $\mathrm{~B}_{1}$ \\
\hline 1842 & $\mathrm{w}$ & $\omega_{3}+\omega_{2}$ & $\mathbf{A}_{1}$ \\
\hline 1923 & vw & $\omega_{3}+\omega_{9}$ & $\mathrm{~B}_{1}$ \\
\hline 2062 & $\mathrm{~m}$ & $2 \omega_{5}$ & $\mathbf{A}_{1}$ \\
\hline & m & $\omega_{3}+\omega_{7}$ & $\mathrm{~B}_{2}$ \\
\hline 2132 & $\mathrm{~m}$ & $\omega_{5}+\omega_{8}$ & $\mathrm{~B}_{2}$ \\
\hline 2204 & $\mathrm{~m}$ & $2 \omega_{8}$ & $\mathbf{A}_{1}$ \\
\hline 2390 & $\mathrm{vw}$ & $\omega_{3}+\omega_{5}$ & $\mathrm{~A}_{2}$ \\
\hline 2456 & $\mathrm{~m}$ & $\omega_{3}+\omega_{8}$ & $\mathrm{~B}_{1}$ \\
\hline 2551 & vw & $\omega_{6}-\omega_{2}$ & $\mathrm{~B}_{2}$ \\
\hline 2850 & w & $\omega_{1}-\omega_{4}$ & $\mathrm{~A}_{1}$ \\
\hline 2967 & $\mathrm{~s}$ & $\omega_{1}$ & $\mathbf{A}_{1}$ \\
\hline 3049 & vs & $\omega_{6}$ & $\mathrm{~B}_{2}$ \\
\hline 3450 & vw & $\omega_{1}+\omega_{2}$ & $\mathbf{A}_{1}$ \\
\hline 3759 & $\mathrm{~m}$ & $\omega_{6}+\omega_{7}$ & $\mathbf{A}_{1}$ \\
\hline 4065 & $\mathrm{~m}$ & $\omega_{1}+\omega_{8}$ & $\mathrm{~B}_{1}$ \\
\hline 4000 & $\mathrm{~m}$ & $\omega_{6}+\omega_{5}$ & $\mathrm{~B}_{1}$ \\
\hline 4397 & $\mathrm{~m}$ & $\omega_{3}+\omega_{6}$ & $\mathrm{~B}_{2}$ \\
\hline
\end{tabular}


TABLE 7. Symmetry effects in the vibrations of molecules of the type $\mathrm{C}-X_{1} X_{2} X_{3} X_{4}$ having tetrahedral symmetry when $X_{1}=X_{2}=X_{3}=X_{4}$

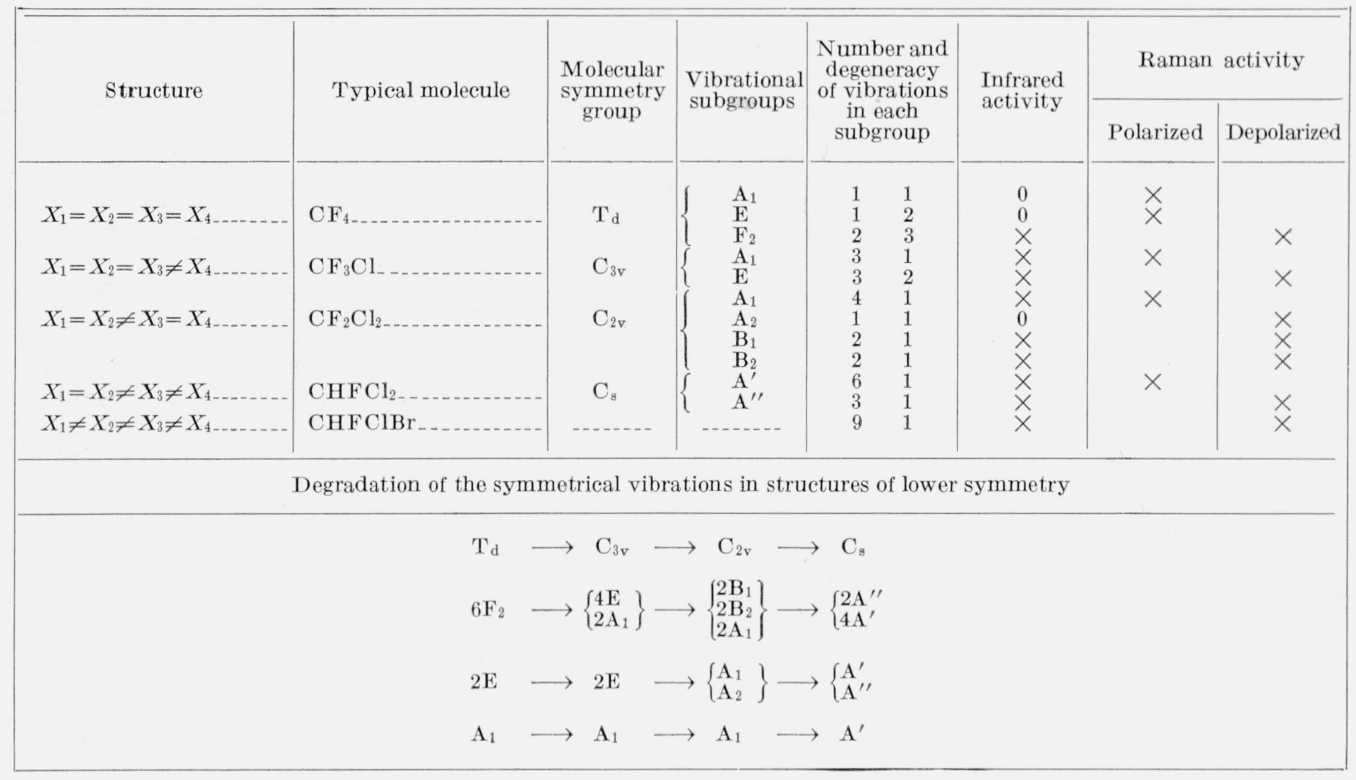

TABLE 8. Fundamental frequencies of methane derivatives, numbered and characterized in different ways

\begin{tabular}{|c|c|c|c|c|c|c|c|c|c|c|c|c|c|}
\hline \multirow{2}{*}{ Conventional No. } & \multicolumn{13}{|c|}{ Methane derivative } \\
\hline & $\mathrm{CF}_{4}$ & $\mathrm{CF}_{3} \mathrm{Cl}$ & $\mathrm{CF}_{2} \mathrm{Cl}_{2}$ & $\mathrm{CFCl}_{3}$ & $\mathrm{CCl}_{4}$ & $\mathrm{CCl}_{3} \mathrm{Br}$ & $\mathrm{CCl}_{2} \mathrm{Br}_{2}$ & $\mathrm{CClBr}_{3}$ & $\mathrm{CBr}_{4}$ & $\mathrm{CHF}_{3}$ & $\mathrm{CHF}_{2} \mathrm{Cl}$ & $\mathrm{CHFCl}_{2}$ & $\mathrm{CHCl}_{3}$ \\
\hline $\int \nu, \mathrm{cm}^{-1} \ldots \ldots$ & 904 & 1105 & 1101 & 1085 & 458 & 719 & 733 & 747 & 267 & 3031 & 3023 & 3023 & 3040 \\
\hline 1 Adopted No & 3 & 1 & 1 & 1 & 3 & 1 & 1 & 1 & 3 & 1 & 1 & 1 & 1 \\
\hline |Character....... & $\nu_{\mathrm{F}, \mathrm{s}}$ & $\nu_{\mathrm{F}, \mathrm{s}}$ & $\nu_{\mathrm{F}, \mathrm{B}}$ & $\nu_{\mathrm{F}}$ & $\nu_{\mathrm{C} 1,8}$ & $\nu_{\mathrm{Cl}, \mathrm{s}}$ & $\nu \mathrm{C} 1.8$ & $\nu \mathrm{Cl}$ & $\nu_{\mathrm{Br}, \mathrm{s}}$ & $\nu_{\mathrm{H}}$ & $\nu_{\mathrm{H}}$ & $\nu_{\mathrm{H}}$ & $\nu_{\mathrm{H}}$ \\
\hline $\int \nu, \mathrm{cm}^{-1} \ldots \ldots$ & 437 & 781 & 667 & 535 & 218 & 420 & 377 & 329 & 123 & 1150 & 1311 & 1304 & 667 \\
\hline $2\{$ Adopted No & 25 & 3 & 3 & 3 & 25 & 3 & 3 & 3 & 25 & 3 & 6 & 6 & 3 \\
\hline Character........ & $\delta_{\mathrm{F}}$ & $\nu_{\mathrm{Cl}}$ & $\nu_{\mathrm{Cl}, \mathrm{s}}$ & $\nu \mathrm{Cl}, \mathrm{B}$ & $\delta_{\mathrm{Cl} 1}$ & $\nu_{\mathrm{Br}}$ & $\nu_{\mathrm{Br}, \mathrm{s}}$ & $\nu_{\mathrm{Br}, \mathrm{B}}$ & $\delta_{\mathrm{Br}}$ & $\nu_{\mathrm{F}, \mathrm{s}}$ & $\delta_{\mathrm{H}}$ & $\delta_{\mathrm{H}}$ & $\nu_{\mathrm{Cl}, \mathrm{s}}$ \\
\hline $\int \nu, \mathrm{cm}^{-1} \ldots \ldots$ & 1277 & 470 & 455 & 350 & 773 & 243 & 252 & 215 & 669 & 700 & 1178 & 1079 & 370 \\
\hline $3\{$ Adopted No & 168 & 4 & 4 & 4 & 168 & 4 & 4 & 4 & 168 & 4 & 3 & 7 & 4 \\
\hline | Character ....... & $\nu_{\mathrm{F}, \mathrm{s}}$ & $\delta_{\mathrm{F}, \mathrm{s}}$ & $\delta_{\mathrm{F}, \mathrm{s}}$ & $\delta \mathrm{Cl}, \mathrm{s}$ & $\nu_{\mathrm{Cl}, \mathrm{a}}$ & $\delta_{\mathrm{Cl}, \mathrm{s}}$ & $\delta \mathrm{Cl}, \mathrm{s}$ & $\delta \delta_{\mathrm{Br}, \mathrm{s}}$ & $\nu_{\mathrm{Br}, \mathrm{a}}$ & $\delta_{\mathrm{F}, \mathrm{A}}$ & $\nu_{F, B}$ & $\nu_{\mathrm{F}}$ & $\delta \mathrm{Cl}, 8$ \\
\hline $\int \nu, \mathrm{cm}^{-1} \ldots$ & 630 & 1212 & 261 & 847 & 319 & 773 & 141 & 675 & 183 & 1372 & 809 & 742 & 1214 \\
\hline $4\{$ Adopted No & 479 & 68 & 2 & 68 & 479 & 68 & 2 & 68 & 479 & 68 & 7 & 3 & 68 \\
\hline lCharacter........ & $\delta_{\mathrm{F}, \mathrm{a}}$ & $\nu_{\mathrm{F}, \mathrm{a}}$ & $\delta \mathrm{Cl}, \mathrm{s}$ & $\nu_{\mathrm{Cl}, \mathrm{a}}$ & $\delta \mathrm{Cl}, \mathrm{a}$ & $\nu_{\mathrm{Cl}, \mathrm{s}}$ & $\delta_{\mathrm{Br}, 8}$ & $\nu_{\mathrm{Br}, \mathrm{a}}$ & $\delta_{\mathrm{Br}, \mathrm{a}}$ & $\nu_{\mathrm{F}, \mathrm{a}}$ & $\nu_{\mathrm{Cl}}$ & $\nu_{\mathrm{Cl}, \mathrm{s}}$ & $\delta_{\mathrm{H}}$ \\
\hline $\int \nu, \mathrm{cm}^{-1} \ldots$ & (n. & 563 & 318 & 401 & $\ldots$ & 294 & 164 & 214 & - & 1152 & 595 & 458 & 755 \\
\hline $5\{$ Adopted No ...... & (2........ & 79 & 5 & 79 & & 79 & 5 & 79 & $\ldots$ & 79 & 4 & 4 & 79 \\
\hline |Character....... & (2........ & $\delta_{\mathrm{F}, \mathrm{a}}$ & $\delta$ & $\delta_{\mathrm{F}}$ & $\cdots$ & $\delta_{\mathrm{C} 1, \mathrm{a}}$ & $\delta$ & $\delta_{\mathrm{Cl}}$ & & $\delta \mathrm{H}$ & $\delta_{\mathrm{F}, \mathrm{s}}$ & $\delta_{\mathrm{F}}$ & $\nu \mathrm{Cl}, \mathrm{s}$ \\
\hline $\int \nu, \mathrm{cm}^{-1} \ldots$ & (n) & 356 & 906 & 248 & & 187 & 768 & 141 & & 507 & 422 & 276 & 261 \\
\hline $6\{$ Adopted No & (n) & 25 & 6 & 25 & & 25 & 6 & 25 & & 25 & 2 & 2 & 25 \\
\hline Character....... & (2....... & $\delta \mathrm{Cl}$ & $\nu \mathrm{Cl}, \mathrm{a}$ & $\delta \mathrm{Cl}, \mathrm{a}$ & & $\delta_{\mathrm{Br}}$ & $\nu_{\mathrm{Cl}, \mathrm{a}}$ & $\delta_{\mathrm{Br}, \mathrm{a}}$ & & $\delta_{\mathrm{F}, \mathrm{a}}$ & $\delta_{\mathrm{Cl}}$ & $\delta \mathrm{Cl}, \mathrm{s}$ & $\delta \mathrm{Cl}, \mathrm{a}$ \\
\hline $\int \nu, \mathrm{cm}^{-1} \ldots \ldots$ & - n & $\ldots$ & 437 & & & . & 252 & & & & 1347 & 1242 & (...... \\
\hline $7\{$ Adopted No & (n. & - & 7 & - & $\ldots$ & -... & 7 & & & & 8 & 8 & - \\
\hline |Character ...... & (2....... & (n) & $\delta \mathrm{FCl}$ & (n. & & - & $\delta \mathrm{Cl}, \mathrm{a}$ & & & & $\delta_{\mathrm{H}}$ & $\delta_{\mathrm{H}}$ & - \\
\hline$\left\{\nu, \mathrm{cm}^{-1} \ldots \ldots\right.$ & (n. & - & 1159 & & & $\cdots$ & 683 & & & & 1116 & 804 & - \\
\hline 8 Adopted No & (n) & (n) & 8 & - & ..... & (2...... & 8 & & & & 9 & 9 & - \\
\hline Char acter....... & (2...... & $\ldots$ & $\nu_{\mathrm{F}, \mathrm{a}}$ & - & $\ldots$ & - & $\nu_{\mathrm{Br}, \mathrm{B}}$ & & & & $\nu_{\mathrm{F}, \mathrm{a}}$ & $\nu_{\mathrm{Cl}, \mathrm{a}}$ & -......... \\
\hline $\int \nu, \mathrm{cm}^{-1} \ldots$ & (n............ & (2) & 473 & (n) & & (n) & 230 & & & & 365 & 368 & (........ \\
\hline $9\{$ Adopted No & (n............ & $\cdots$ & 9 & (n) & ... & $\cdots$ & 9 & - n & $\cdots$ & $\ldots$ & 5 & 5 & -.......... \\
\hline |Character........ & . & & $\delta \mathrm{FCl}$ & $\cdots$ & & 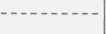 & $\delta_{\mathrm{Br}, \mathrm{a}}$ & $\cdots$ & & $\cdots$ & $\delta \mathrm{FCl}$ & $\delta \mathrm{FCl}$ & -......... \\
\hline
\end{tabular}


TABLE 8. Fundamental frequencies of menthane derivatives, numbered and characterized in different ways-Continued

\begin{tabular}{|c|c|c|c|c|c|c|c|c|c|c|c|c|}
\hline \multirow{2}{*}{ Conventional No. } & \multicolumn{12}{|c|}{ Methane derivative } \\
\hline & $\mathrm{CHCl}_{2} \mathrm{Br}$ & $\mathrm{CHClBr}_{2}$ & $\mathrm{CHBr}{ }_{3}$ & $\mathrm{CHI}_{3}$ & $\mathrm{CH}_{2} \mathrm{~F}_{2}$ & $\mathrm{CH}_{2} \mathrm{ClF}$ & $\mathrm{CH}_{2} \mathrm{Cl}_{2}$ & $\mathrm{CH}_{2} \mathrm{ClBr}$ & $\mathrm{CH}_{2} \mathrm{Br}_{2}$ & $\mathrm{CH}_{2} \mathrm{I}_{2}$ & $\mathrm{CH}_{3} \mathrm{~F}$ & $\mathrm{CH}_{4}$ \\
\hline $\int \nu, \mathrm{cm}^{-1} \ldots \ldots$ & 3040 & 3034 & 3040 & $(3040)$ & 2949 & 2993 & 2985 & 2987 & 2988 & 2967 & 2964 & 2915 \\
\hline 1 Adopted No... & 1 & 1 & 1 & 1 & 1 & 1 & 1 & 1 & 1 & 1 & 1 & 3 \\
\hline | Character..... & $\nu_{\mathrm{H}}$ & $\nu_{\mathrm{H}}$ & $\nu_{\mathrm{H}}$ & $\nu_{\mathrm{H}}$ & $\nu_{\mathrm{H}, \mathrm{s}}$ & $\nu_{\mathrm{H}, \mathrm{s}}$ & $\nu_{\mathrm{H}, \mathrm{s}}$ & $\nu_{\mathrm{H}, \mathrm{s}}$ & $\nu_{\mathrm{H}, \mathrm{s}}$ & $\nu_{\mathrm{H}, \mathrm{s}}$ & $\nu_{\mathrm{H}, \mathrm{s}}$ & $\nu_{\mathrm{H}, \mathrm{s}}$ \\
\hline $\int \nu, \mathrm{cm}^{-1} \ldots \ldots$ & 1168 & 1189 & 541 & 385 & 1508 & 1470 & 1424 & 1402 & 1385 & 1351 & 1475 & 1533 \\
\hline $2\{$ Adopted No.... & 6 & 6 & 3 & 3 & 3 & 3 & 3 & 3 & 3 & 3 & 3 & 25 \\
\hline Character_...... & $\delta_{\mathrm{H}}$ & $\delta_{\mathrm{H}}$ & $\nu \mathrm{Br}, \mathrm{s}$ & $\nu_{\mathrm{I}, \mathrm{s}}$ & $\delta_{\mathrm{H}, \mathrm{s}}$ & $\delta_{\mathrm{H}, \mathrm{s}}$ & $\delta_{\mathrm{H}, \mathrm{B}}$ & $\delta_{\mathrm{H}, \mathrm{s}}$ & $\delta_{\mathrm{H}, \mathrm{s}}$ & $\delta_{\mathrm{H}, \mathrm{s}}$ & $\delta_{\mathrm{H}, \mathrm{s}}$ & $\delta_{\mathrm{H}}$ \\
\hline $\int \nu, \mathrm{cm}^{-1} \ldots \ldots$ & 719 & 746 & 222 & 145 & 1116 & 1351 & 706 & 1225 & 579 & 486 & 1048 & 3020 \\
\hline $3\{$ Adopted No & 7 & 7 & 4 & 4 & 2 & 8 & 2 & 8 & 2 & 2 & 4 & 168 \\
\hline |Character...... & $\nu \mathrm{Cl}, 8$ & $\nu_{\mathrm{Cl}}$ & $\delta_{\mathrm{Br}, 8}$ & $\delta_{\mathrm{I}, \mathrm{B}}$ & $\nu_{\mathrm{F}, 8}$ & $\delta_{\mathrm{HF}}$ & $\nu_{\mathrm{Cl} 1, \mathrm{~s}}$ & $\delta \mathrm{HCl}$ & $\nu_{\mathrm{Br}, \mathrm{s}}$ & $\nu_{1,8}$ & $\nu_{\mathrm{F}}$ & $\nu_{\mathrm{H}, \mathrm{s}}$ \\
\hline $\int \nu, \mathrm{cm}^{-1} \ldots \ldots$ & 604 & 570 & 1142 & 1064 & 529 & 1068 & 286 & 728 & 174 & 128 & 2982 & 1304 \\
\hline 4 Adopted No & 3 & 3 & 68 & 68 & 4 & 7 & 4 & 9 & 4 & 4 & 68 & 479 \\
\hline |Character....... & $\nu_{\mathrm{Br}}$ & $\nu \mathrm{Br}, \mathrm{s}$ & $\delta_{\mathrm{H}}$ & $\delta_{\mathrm{H}}$ & $\delta_{\mathrm{F}, \mathrm{s}}$ & $\nu_{\mathrm{F}}$ & $\delta_{\mathrm{Cl}, \mathrm{s}}$ & $\nu_{\mathrm{Cl}}$ & $\delta_{\mathrm{Br}}$ & $\delta_{\mathrm{I}}$ & $\nu_{\mathrm{H}, \mathrm{s}}$ & $\delta_{\mathrm{H}, \mathrm{s}}$ \\
\hline$\nu, \mathrm{cm}^{-1} \ldots$ & 330 & 278 & 668 & 581 & 1262 & 760 & 1159 & 606 & 1096 & 1035 & 1471 & \\
\hline $5\{$ Adopted No....... & 4 & 4 & 79 & 79 & 5 & 2 & 5 & 2 & 5 & 5 & 79 & \\
\hline |Character & $\delta \mathrm{C} 1, \mathrm{~B}$ & $\delta_{\mathrm{C} 1}$ & $\nu_{\mathrm{Br}, \mathrm{a}}$ & $\nu_{\mathrm{I}, \mathrm{a}}$ & $\delta$ & $\nu_{\mathrm{C} 1}$ & $\delta$ & $\nu_{\mathrm{Br}}$ & $\delta$ & $\delta$ & $\delta_{\mathrm{H}, \mathrm{s}}$ & \\
\hline $\int \nu, \mathrm{cm}^{-1} \ldots$ & 220 & 168 & 154 & 92 & 3012 & 385 & 3048 & 226 & 3065 & 3049 & 1196 & \\
\hline 6 Adopted No_..... & 2 & 2 & 25 & 25 & 6 & 4 & 6 & 4 & 6 & 6 & 25 & \\
\hline |Character & $\delta_{\mathrm{Br}}$ & $\delta_{\mathrm{Br}, \mathrm{s}}$ & $\delta_{\mathrm{Br}, \mathrm{a}}$ & $\delta_{\mathrm{I}, \mathrm{a}}$ & $\nu_{\mathrm{H}, \mathrm{a}}$ & $\delta_{\mathrm{Cl}}$ & $\nu_{\mathrm{H}, \mathrm{a}}$ & $\delta_{\mathrm{Br}}$ & $\nu \mathrm{H}, \mathrm{s}$ & $\nu_{\mathrm{H}, \mathrm{a}}$ & $\delta_{\mathrm{HF}}$ & \\
\hline$\nu, \mathrm{em}^{-1} \ldots$ & 1211 & 1144 & & & 1176 & 3048 & 898 & 3060 & 813 & 717 & & \\
\hline $7\{$ Adopted No_...... & 8 & 8 & & & 7 & 6 & 7 & 6 & 7 & 7 & & \\
\hline |Character-_...... & $\delta_{\mathrm{H}}$ & $\delta_{\mathrm{H}}$ & & & $\delta_{\mathrm{HF}}$ & $\nu_{\mathrm{H}, \mathrm{a}}$ & $\delta_{\mathrm{HCl}}$ & $\nu_{\mathrm{H}, \mathrm{a}}$ & $\delta \mathrm{H} \mathrm{Br}$ & $\delta_{\mathrm{HI}}$ & & \\
\hline $\int \nu, \mathrm{cm}^{-1} \ldots$ & 760 & 657 & & & 1435 & 1236 & 1262 & 1130 & 1190 & 1107 & & \\
\hline 8 Adopted No & 9 & 9 & & & 8 & 5 & 8 & 5 & 8 & 8 & & \\
\hline |Character-_. & $\nu_{\mathrm{Cl}, \mathrm{a}}$ & $\nu_{\mathrm{Br}, \mathrm{a}}$ & & & $\delta_{\mathrm{HF}}$ & $\delta_{\mathrm{HF}}$ & $\delta \mathrm{HCl}$ & $\delta_{\mathrm{HC} 1}$ & $\delta_{\mathrm{HBr}}$ & $\delta_{\mathrm{HI}}$ & & \\
\hline $\int \nu, \mathrm{cm}^{-1} \ldots \ldots \ldots$ & 215 & 201 & & & 1090 & 1004 & 742 & 852 & 639 & 572 & & \\
\hline $9\{$ Adopted No_............. & 5 & 5 & & & 9 & 9 & 9 & 7 & 9 & 9 & & \\
\hline |Character & $\delta \mathrm{ClBr}$ & $\delta_{\mathrm{ClBr}}$ & & & $\nu_{\mathrm{F}, \mathrm{a}}$ & $\delta \mathrm{HCl}$ & $\nu_{\mathrm{Cl}, \mathrm{a}}$ & $\delta \mathrm{HBr}$ & $\nu \mathrm{Br}, \mathrm{a}$ & $\nu_{\mathrm{I}, \mathrm{a}}$ & & \\
\hline
\end{tabular}

The regularities mentioned in the preceding paragraph are displayed in figures 13 to 15 , inclusive. In these graphs the frequencies of related molecules are plotted, the molecules being arranged vertically in order of increasing molecular weight. The regular decrease in frequency with increasing weight of the substituent, and the way in which the degenerate frequencies in the more symmetrical molecules break up in the less symmetrical ones and then regroup, is to be noted.

Another often-used method of identifying the fundamentals, which also possesses a degree of physical significance, is to characterize them by the type of intramolecular motion involved. In this method, $\nu_{\mathrm{x}}$ signifies the stretching of a bond connecting the carbon atom with the $\mathrm{x}$ substituent, and $\delta_{\mathrm{x}}$ signifies the bending of such a bond. All the molecules considered will have four $\nu$ 's and five $\delta$ 's. When there are several identical bonds, the suffix $s$ or $a$ may be added, according to whether the motion of the identical atoms is symmetrically toward the central atom or otherwise. In molecules as light as those considered here, this method often has limited meaning, since the motions in each fundamental mode cannot be localized as closely as the method would imply; in the $\delta$ modes in particular all the atoms participate in the motion. Nevertheless, a characterization of the fundamentals by this method is also included in table 8 and will be referred to in later discussion. For some such cases no suffix can be given; in others, a double suffix identifies the two atoms most prominently involved.
The relative intensity of the various fundamentals is not displayed in figures 13 to 15 . The intensity depends on a number of factors, principally the symmetry of the motion and the distribution of electrical charge within the molecule. From the spectral graphs and tables it will be noted that there is a general parallelism in the intensities of corresponding bands in the various molecules of like symmetry. It should be noted that, although for molecules of symmetry $\mathrm{C}_{2 \mathrm{v}}$, the $\mathrm{A}_{2}$ frequency $\omega_{5}$ should be inactive, it does appear in all the molecules considered, if it falls in the observable frequency range. It is always quite weak, however, and its appearance may readily be explained as due to a breakdown of the strict selection rules because of intermolecular forces, particularly in the liquid state, or to Coriolis interactions with nearby active frequencies, or to a combination of these causes.

\subsection{Force Constant Calculations}

For a quantitative verification of the assignment of fundamentals, recourse must be had to a calculation in terms of the atomic masses, atomic dimensions, and a suitable set of force constants of the potential energy. In the most general potential function there are many more disposable constants than there are observed frequencies, so that various approximations must be introduced. Certain of the constants, especially those involving interactions between motions of different types, may be set equal to zero; other constants may be assumed to have the 
same numerical value in different molecules. Many such treatments have been reported for most of the molecules considered here. One of the more successful of the recent simpler calculations was that of Decius [5], who was able to calculate 82 frequencies in 14 normal and deuterated chloro-bromo-methanes from 28 constants, with a mean accuracy of 1.1 percent.

In order to substantiate some of our assignments, and to determine whether a similar treatment may be applied to compounds containing fluorine, the calculations of Decius have been extended to the molecules $\mathrm{CF}_{4}, \mathrm{CF}_{3} \mathrm{H}, \mathrm{CF}_{2} \mathrm{H}_{2}, \mathrm{CFH}_{3}, \mathrm{CF}_{3} \mathrm{Cl}, \mathrm{CF}_{2} \mathrm{Cl}_{2}$, $\mathrm{CFCl}_{3}, \mathrm{CCl}_{3} \mathrm{Br}, \mathrm{CCl}_{2} \mathrm{Br}_{2}$, and $\mathrm{CClBr}_{3}$, for which all nine frequencies were computed; and to the less symmetrical molecules $\mathrm{CHF}_{2} \mathrm{Cl}, \mathrm{CHFCl}_{2}, \mathrm{CHCl}_{2} \mathrm{Br}$, $\mathrm{CHClBr}_{2}, \mathrm{CH}_{2} \mathrm{FCl}$, and $\mathrm{CH}_{2} \mathrm{ClBr}$, for which only the three frequencies of symmetry $\mathrm{A}^{\prime \prime}$ were computed. (To evaluate the remaining six frequencies of the latter molecules would involve the expansion and solution of a sixth-order determinant.) In these calculations the constants and molecular dimensions of Decius were adopted when applicable (one apparent numerical error or misprint, for $f_{\beta}^{1}$ in $\mathrm{C}-\mathrm{Cl}$, was corrected). New values for the corresponding constants involving fluorine were adopted, which gave a fair over-all fit of the data considered. It is probable that additional computations would yield somewhat better values of these constants, but this does not seem useful, in view of the approximations involved. The constants used are listed in Decius' notation in table 9. Calculations on "mixed" molecules, that is, those containing more than one halogen, require additional constants. For these are used the geometric mean of the corresponding

TABLE 9.-Force constants for halomethanes (after Decius)

The following constants are assumed to be zero: $f_{R r}^{1}, f_{R \eta}^{1}, f_{r}^{2}, f_{r \alpha}^{1}, f_{r \alpha}^{2}, f_{r \beta}^{1}, f_{r \eta}^{1}$, $f_{F \eta}^{2}, f_{\alpha \beta}^{1}, f_{\alpha \beta}^{2}, f_{\beta \eta}^{2}, f_{\eta}^{2}$, and $f_{\eta}^{3}$.

The numerical units are $10^{5}$ dyne $\mathrm{cm}^{-1}$ for two stretching coordinates, $10^{-3}$ dyne for bending and stretching, and $10^{-11} \mathrm{dyne} \mathrm{cm}$ for two bending coordinates

$r_{0} \mathrm{C}-\mathrm{F}=1.35 \quad \mathrm{~A}, r_{0} \mathrm{C}-\mathrm{Cl}=1.75 \quad \mathrm{~A}, r_{0} \mathrm{C}-\mathrm{Br}=1.88 \quad \mathrm{~A}, r_{0} \mathrm{C}-\mathrm{H}=1.093 \quad \mathrm{~A}$, al $\alpha_{0}=\boldsymbol{\beta}_{0}=\eta_{0}=109^{\circ} 28^{\prime}$

\begin{tabular}{|l|l|c|c|c|}
\hline \hline Constant & \multicolumn{1}{|c|}{ Terms involved } & $\mathrm{X}^{\mathrm{i}}=\mathrm{F}$ & $\mathrm{X}^{\mathrm{i}}=\mathrm{Cl}$ & $\mathrm{X}^{\mathrm{i}}=\mathrm{Br}$ \\
\hline$f_{\mathrm{R}}^{1}$ & $\mathrm{C}-\mathrm{X}_{\mathrm{j}}^{\mathrm{i}} \cdot \mathrm{C}-\mathrm{X}_{\mathrm{j}}^{\mathrm{i}}$ & 6.26 & 3.383 & 2.840 \\
$f_{\mathrm{R}}^{2}$ & $\mathrm{C}-\mathrm{X}_{\mathrm{j}}^{\mathrm{i}} \cdot \mathrm{C}-\mathrm{X}_{\mathrm{k}}^{\mathrm{i}}$ & 0.96 & 0.332 & 0.186 \\
$f_{r}^{1}$ & $\mathrm{C}-\mathrm{H}_{\mathrm{i}} \cdot \mathrm{C}-\mathrm{H}_{\mathrm{i}}$ & 5.04 & 5.04 & 5.04 \\
$f_{\mathrm{R} \alpha}^{1}$ & $\mathrm{C}-\mathrm{X}_{\mathrm{j}}^{\mathrm{i}} \cdot \mathrm{H}-\mathrm{C}-\mathrm{X}_{\mathrm{j}}^{\mathrm{i}}$ & 0.40 & 0.340 & 0.305 \\
$f_{\mathrm{R} \alpha}^{2}$ & $\mathrm{C}-\mathrm{X}_{\mathrm{j}}^{\mathrm{i}} \cdot \mathrm{H}-\mathrm{C}-\mathrm{X}_{\mathrm{k}}^{\mathrm{i}}$ & -.21 & -.164 & -.152 \\
$f_{\mathrm{R} \beta}^{1}$ & $\mathrm{C}-\mathrm{X}_{\mathrm{j}}^{\mathrm{i}} \cdot \mathrm{X}_{\mathrm{j}}^{\mathrm{i}}-\mathrm{C}-\mathrm{X}_{\mathrm{k}}^{\mathrm{i}}$ & .34 & .338 & .342 \\
$f_{\mathrm{R} \beta}^{2}$ & $\mathrm{C}-\mathrm{X}_{\mathrm{j}}^{\mathrm{i}} \cdot \mathrm{X}_{\mathrm{k}}^{\mathrm{i}}-\mathrm{C}-\mathrm{X}_{\mathrm{i}}^{\mathrm{i}}$ & -.34 & -.249 & -.190 \\
$f_{\alpha}^{1}$ & $\mathrm{H}_{\mathrm{j}}-\mathrm{C}-\mathrm{X}_{\mathrm{j}}^{\mathrm{i}} \cdot \mathrm{H}_{\mathrm{j}}-\mathrm{C}-\mathrm{X}_{\mathrm{j}}^{\mathrm{i}}$ & .875 & .687 & .589 \\
$f_{\alpha}^{2}$ & $\mathrm{H}_{\mathrm{j}}-\mathrm{C}-\mathrm{X}_{\mathrm{j}}^{\mathrm{i}} \cdot \mathrm{H}_{\mathrm{j}}-\mathrm{C}-\mathrm{X}_{\mathrm{k}}^{\mathrm{i}}$ & .050 & .009 & -.005 \\
$f_{\alpha}^{3}$ & $\mathrm{H}_{\mathrm{j}}-\mathrm{C}-\mathrm{X}_{\mathrm{j}}^{\mathrm{i}} \cdot \mathrm{H}_{\mathrm{k}}-\mathrm{C}-\mathrm{X}_{\mathrm{j}}^{\mathrm{i}}$ & -.048 & -.034 & -.026 \\
$f_{\alpha}^{4}$ & $\mathrm{H}_{\mathrm{j}}-\mathrm{C}-\mathrm{X}_{\mathrm{j}}^{\mathrm{i}} \cdot \mathrm{H}_{\mathrm{k}}-\mathrm{C}-\mathrm{X}_{\mathrm{k}}^{\mathrm{i}}$ & -.145 & -.065 & -.060 \\
$f_{\beta}^{1}$ & $\mathrm{X}_{\mathrm{j}}^{\mathrm{i}}-\mathrm{C}-\mathrm{X}_{\mathrm{k}}^{\mathrm{i}} \cdot \mathrm{X}_{\mathrm{j}}^{\mathrm{i}}-\mathrm{C}-\mathrm{X}_{\mathrm{k}}^{\mathrm{i}}$ & 1.75 & 1.236 & 1.061 \\
$f_{\beta}^{2}$ & $\mathrm{X}_{\mathrm{j}}^{\mathrm{i}}-\mathrm{C}-\mathrm{X}_{\mathrm{k}}^{\mathrm{i}} \cdot \mathrm{X}_{\mathrm{j}}^{\mathrm{i}}-\mathrm{C}-\mathrm{X}_{\mathrm{l}}^{\mathrm{i}}$ & 0.17 & 0.092 & 0.134 \\
$f^{3}$ & $\mathrm{X}_{\mathrm{j}}^{\mathrm{i}}-\mathrm{C}-\mathrm{X}_{\mathrm{k}}^{\mathrm{i}} \cdot \mathrm{X}_{\mathrm{i}}^{\mathrm{i}}-\mathrm{C}-\mathrm{X}_{\mathrm{m}}^{\mathrm{i}}$ & -.10 & -.037 & .039 \\
$f_{\eta}^{1}$ & $\mathrm{H}_{\mathrm{j}}-\mathrm{C}-\mathrm{H}_{\mathrm{k}} \cdot \mathrm{H}_{\mathrm{j}}-\mathrm{C}-\mathrm{H}_{\mathrm{k}}$ & .530 & .530 & .530 \\
\hline
\end{tabular}

constants involving a single halogen. For example, since the constant $f_{\mathrm{C}-\mathrm{x}}^{2}$, corresponding to the interaction of motion along two $\mathrm{C}-\mathrm{X}^{1}$ bonds, $\mathrm{C}-\mathrm{X}_{\mathrm{j}}^{\mathrm{i}} \cdot \mathrm{C}-\mathrm{X}_{\mathrm{k}}^{\mathrm{i}}$, is 0.96 (in units of $10^{5} \mathrm{dyne} / \mathrm{cm}$ ) for $\mathrm{X}^{1}=\mathrm{F}$ and 0.332 for $\mathrm{X}^{1}=\mathrm{Cl}$, the constant for interaction of motion along $\mathrm{C}-\mathrm{F}$ and $\mathrm{C}-\mathrm{Cl}$ would be $[(0.96) \cdot(0.332)]^{1 / 2}=0.565$. The constants involving

TABLE 10. Comparison of calculated and observed frequencies $\left(\Delta=\frac{100\left(\nu_{\mathrm{obs}}-\nu_{\mathrm{ealc}}\right)}{\nu_{\mathrm{obs}}}\right)$

\begin{tabular}{|c|c|c|c|c|c|}
\hline$\omega$ & $\nu_{\text {osle }}$ & $\Delta$ & $\omega$ & $\nu_{\text {calce }}$ & $\Delta$ \\
\hline \multicolumn{3}{|c|}{$\mathrm{CF}_{4}$} & \multicolumn{3}{|c|}{$\mathrm{CF}_{3} \mathrm{H}$} \\
\hline 3 & 904 & 0.0 & \multirow{5}{*}{$\begin{array}{r}1 \\
3 \\
4 \\
68 \\
79 \\
25\end{array}$} & \multirow{5}{*}{$\begin{array}{r}3047 \\
1139 \\
665 \\
1369 \\
1140 \\
532\end{array}$} & \multirow{5}{*}{$\begin{array}{r}-0.5 \\
1.0 \\
5.0 \\
0.2 \\
-1.2 \\
-4.8 \\
\end{array}$} \\
\hline 25 & 437 & .0 & & & \\
\hline 168 & 1234 & 3.4 & & & \\
\hline 479 & 613 & 2.7 & & & \\
\hline \multicolumn{3}{|c|}{$\mathrm{CF}_{2} \mathrm{H}_{2}$} & & & \\
\hline 1 & \multirow{8}{*}{$\begin{array}{r}3005 \\
1466 \\
1154 \\
537 \\
1262 \\
3082 \\
1108 \\
1475 \\
1150\end{array}$} & \multirow{8}{*}{$\begin{array}{r}1.9 \\
2.8 \\
-3.0 \\
-1.5 \\
0.0 \\
-2.3 \\
5.8 \\
-2.8 \\
-5.5\end{array}$} & \multicolumn{3}{|c|}{$\mathrm{CFH}_{3}$} \\
\hline 3 & & & & & \\
\hline${ }_{2}^{4}$ & & & ${ }_{3}^{1}$ & $\begin{array}{l}2960 \\
1552\end{array}$ & $\begin{array}{l}0.1 \\
1.6\end{array}$ \\
\hline 5 & & & 4 & 1125 & 7. 3 \\
\hline 6 & & & 68 & 3072 & -3.0 \\
\hline 7 & & & 79 & 1479 & 0. 5 \\
\hline 8 & & & 25 & 1181 & \\
\hline 9 & & & & & \\
\hline \multicolumn{3}{|c|}{$\mathrm{CF}_{3} \mathrm{Cl}$} & \multicolumn{3}{|c|}{$\mathrm{CF}_{2} \mathrm{Cl}_{2}$} \\
\hline & & & 1 & 1126 & $\begin{array}{r}-2.3 \\
-8\end{array}$ \\
\hline $\begin{array}{l}1 \\
3\end{array}$ & $\begin{array}{r}1090 \\
744\end{array}$ & $\begin{array}{l}0.8 \\
4.7\end{array}$ & $\begin{array}{l}3 \\
4\end{array}$ & $\begin{array}{l}648 \\
463\end{array}$ & $\begin{array}{r}2.8 \\
-1.8\end{array}$ \\
\hline 4 & 460 & 2. 2 & 2 & 262 & -0.4 \\
\hline 68 & 1191 & 1.8 & 5 & 331 & -4.1 \\
\hline 79 & 562 & 0.2 & 6 & 867 & 4. 3 \\
\hline 25 & 358 & -.5 & \multirow{2}{*}{$\begin{array}{l}7 \\
8 \\
9\end{array}$} & \multirow{2}{*}{$\begin{array}{r}433 \\
1168 \\
460\end{array}$} & \multirow{2}{*}{$\begin{array}{r}0.9 \\
-.8 \\
2.8\end{array}$} \\
\hline \multicolumn{3}{|c|}{$\mathrm{CFCl}_{3}$} & & & \\
\hline 1 & \multirow{5}{*}{$\begin{array}{r}1125 \\
524 \\
363 \\
813 \\
420 \\
243\end{array}$} & \multirow{5}{*}{$\begin{array}{r}-3.7 \\
2.1 \\
-3.7 \\
4.0 \\
-4.8 \\
2.0\end{array}$} & \multicolumn{3}{|c|}{$\mathrm{CCl}_{3} \mathrm{Br}$} \\
\hline $\begin{array}{l}3 \\
4\end{array}$ & & & & & \\
\hline 68 & & & $\begin{array}{l}1 \\
3\end{array}$ & $\begin{array}{l}450 \\
450\end{array}$ & -7.1 \\
\hline 79 & & & 4 & 235 & -3.3 \\
\hline 25 & & & 68 & 768 & 0.7 \\
\hline \multicolumn{3}{|c|}{$\mathrm{CCl}_{2} \mathrm{Br}_{2}$} & 25 & 174 & 6.9 \\
\hline 1 & 734 & & \multicolumn{3}{|c|}{$\mathrm{CBr}_{3} \mathrm{Cl}$} \\
\hline 3 & 364 & 3.5 & & & \\
\hline${ }_{2}^{4}$ & $\begin{array}{l}251 \\
147\end{array}$ & $\begin{array}{r}0.4 \\
-4.3\end{array}$ & 1 & 751 & -0.5 \\
\hline$\frac{2}{5}$ & $\begin{array}{l}147 \\
168\end{array}$ & $\begin{array}{l}-4.3 \\
-2.5\end{array}$ & ${ }_{4}^{3}$ & $\begin{array}{l}324 \\
215\end{array}$ & $\begin{array}{l}1.6 \\
0.0\end{array}$ \\
\hline 6 & 772 & -0.5 & 68 & 678 & -.5 \\
\hline 7 & 260 & -3.2 & 79 & 21.5 & -.4 \\
\hline 8 & 676 & 1.0 & 25 & 137 & 2.8 \\
\hline 9 & 229 & 0.4 & & & \\
\hline & $\mathrm{CHF}_{2} \mathrm{C}$ & & & $\mathrm{CHBr}_{2}$ & \\
\hline 8 & 1366 & & 8 & & 0.8 \\
\hline 9 & 1132 & $\begin{array}{l}-1.4 \\
-1.4\end{array}$ & 9 & $\begin{array}{l}651 \\
205\end{array}$ & $\begin{array}{r}.9 \\
-20\end{array}$ \\
\hline 5 & 391 & -7.1 & & & \\
\hline & $\mathrm{CHFC}$ & & & $\mathrm{CH}_{2} \mathrm{FC}$ & \\
\hline & 1221 & & & & \\
\hline 9 & 791 & 1.6 & $\begin{array}{l}5 \\
0\end{array}$ & 1214 & $\begin{array}{r}1.8 \\
-01\end{array}$ \\
\hline 5 & 392 & & & & \\
\hline & $\mathrm{CHCl}_{2} \mathrm{I}$ & & & $\mathrm{CH}_{2} \mathrm{ClF}$ & \\
\hline 8 & 1221 & -4.4 & 6 & 3077 & \\
\hline 9 & $\begin{array}{l}749 \\
219\end{array}$ & $\begin{array}{r}1.5 \\
1.9\end{array}$ & 5 & 1142 & -1.1 \\
\hline 5 & 219 & -1.9 & 7 & 846 & -0.7 \\
\hline
\end{tabular}


bending of $\mathrm{X}^{1}-\mathrm{C}-\mathrm{X}^{1}$ bonds and their interactions are obtained similarly, without introducing further new constants.

The resulting computed frequencies are compared with the observed fundamentals in table 10 . It is seen that, in general, the agreement is good. The greatest deviation is 7.3 percent, and the average deviation of 85 frequencies in 16 molecules is 2.22 percent. This degree of fit was obtained by introducing 13 new constants, in addition to the 28 taken from Decius. Better agreement is not to be expected in view of the approximations introduced. Inasmuch as the very accurate results of microwave spectroscopy show that the interatomic distances are not constant, particularly for the $\mathrm{C}-\mathrm{F}$ bond, and that the angles are not tetrahedral, it is clear that the force constants must vary to some extent. The interaction constants are relatively large: the particularly high value of $f_{\mathrm{R}}^{2}$ for $\mathrm{C}-\mathrm{F}\left(0.96 \times 10^{5}\right.$ dyne $\mathrm{cm}^{-1}$ ) is to be noted. This is a further reflection of the importance of specific forces arising from the highly polar $\mathrm{C}-\mathrm{F}$ linkage, and indicates that for a truly accurate calculation, one should take into account all the ignored interactions, perhaps basing them on some more general force field [6]. The present comparison serves merely to confirm the choice of fundamentals and to demonstrate the approximate similarity of the carbon-halogen bonds in a large number of halomethanes.

It should be noted that the $\mathrm{C}-\mathrm{F}$ constants, particularly the major ones involving the stretching, are in good agreement with a very recently published calculation based on much the same data and methods [7]. It should also be mentioned that preliminary calculations indicate that the $\mathrm{C}-\mathrm{F}$ constants used also give adequate agreement with observation in such molecules as $\mathrm{C}_{2} \mathrm{~F}_{6}, \mathrm{CF}_{3}-\mathrm{CH}_{3}$, $\mathrm{CF}_{3}-\mathrm{CCl}_{3}$, etc.

There are not enough data on the iodine compounds to derive constants for this bond, but approximate values extrapolated from the series $\mathrm{F} \rightarrow \mathrm{Cl} \rightarrow \mathrm{Br} \rightarrow \mathrm{I}$ may be seen to give adequate agreement with the few observations.

\subsection{Overtone, Combination, and Difference Bands}

In addition to the fundamentals, the bands that appear in the spectrum must be attributed to overtones, combinations, and differences of the fundamentals. They have been so assigned in tables 2 to 6 . The assignments appear eminently reasonable, in that the selection rules are obeyed, in the molecules of higher symmetry, with the exception of an occasional weak combination of symmetry $A_{2}$ in $\mathrm{C}_{2 \mathrm{v}}$; that binary combinations are in general much stronger than those involving three frequencies; and that difference bands arise only from relatively low vibrational frequencies.

Under the dispersion of prism instruments, it is not always possible to make an unambiguous assignment of these higher-order bands. As will be noted in tables 2 to 6 , there are many instances in which two or more combinations can be offered as probable, and many other such have not been listed separately.
The magnitude of the anharmonic corrections, by which the frequencies of the overtones and combinations differ from the sum of the fundamentals, ranges from +10 to $-50 \mathrm{~cm}^{-1}$, and, with nine fundamentals, such overlapping is common. Unless much higher resolution is available, it is unprofitable to attempt to distinguish between the possibilities, except to note that the assignments that are set forth show a consistent behavior in a large number of molecules. Indeed, because of the possibility of Fermi resonance among levels of like symmetry, it is often meaningless to distinguish between closelying levels. Some of the more striking and definite examples of this effect occur in $\mathrm{CCl}_{4}$ and $\mathrm{CCl}_{2} \mathrm{~F}_{2}$ and will be discussed in more detail later. In a very heavy molecule of low symmetry, such as $\mathrm{CCl}_{2} \mathrm{Br}_{2}$, the number of possible difference bands of appreciable intensity arising from the five fundamentals below $300 \mathrm{~cm}^{-1}$ is particularly large. It will be noted in figure 6 that in these molecules there is a rather strong background of structureless absorption throughout the region 280 to $500 \mathrm{~cm}^{-1}$, which undoubtedly arises from such overlappings.

It should be mentioned that a number of the overtone and combination bands of the methylene-halide compounds $\mathrm{CH}_{2} \mathrm{~F}_{2}, \mathrm{CH}_{2} \mathrm{FCl}, \mathrm{CH}_{2} \mathrm{Cl}_{2}, \mathrm{CH}_{2} \mathrm{ClBr}$, and $\mathrm{CH}_{2} \mathrm{Br}_{2}$ have been studied under very high resolving power in the region 1.6 to $2.5 \mu$. The results will be reported separately. They show the expected large number of bands, with the rotational structure partially resolved, and further confirm the vibrational assignments reported in this paper.

\subsection{Liquid-Vapor Shifts}

As mentioned above, a number of the compounds of low boiling point have been studied both as liquids and as vapors, at room temperature. As is commonly observed, the vapor spectra show more indications of partially resolved rotational structure ( $P, D$, and $R$ branches), but otherwise do not differ appreciably from those of the liquids, except for slight shifts of the central absorption frequencies. Some of these shifts are listed in table 11 . It will be noted that the frequencies for the vapor are consistently higher than for the liquid, and that the magnitude of the shift is in general greater, especially on a percentage basis, for the stretching frequencies involving the halogens ( $\nu_{\mathrm{x}}$ 's) than for the bending frequencies or for the hydrogen-stretching frequencies. This is undoubtedly due to the fact that the shifts result from partial association in the liquid, which is predominantly due to forces between the dipoles localized in the carbon-halogen bonds.

The fluorine compounds $\mathrm{CH}_{2} \mathrm{~F}_{2}$ and $\mathrm{CHF}_{3}$ were studied in this research only in the gaseous state. Their Raman spectra have, however, been studied in the liquid. Since the $\mathrm{C}-\mathrm{F}$ bond is considerably more polar than $\mathrm{C}-\mathrm{Cl}$, one might expect the $\nu_{\mathrm{F}}$ frequencies to show quite large liquid-vapor shifts, and these have been observed. These two molecules have also been listed in table 11, the $\Delta$ now referring to the shift between our adopted infrared frequencies and the most recent Raman data [8]. 
TABLE 11. Frequency differences $\left(\nu_{\text {vapor }}-\nu_{\text {li ouid }}\right)$ for some halomethanes

\begin{tabular}{|c|c|c|c|}
\hline Vibration & $\Delta \nu \mathrm{cm}^{-1}$ & Vibration & $\Delta \nu$ \\
\hline \multicolumn{2}{|c|}{$\mathrm{CHCl}_{3}$} & \multicolumn{2}{|c|}{$\mathrm{CHCl}_{2} \mathrm{Br}$} \\
\hline $\begin{array}{l}\omega_{32 \nu \mathrm{C} 1,8} \\
\omega_{70} \nu \mathrm{Cl}, \mathrm{s} \\
\omega_{68} \delta \mathrm{H} \\
\omega_{1} \nu \mathrm{H}\end{array}$ & $\begin{array}{r}+13 \\
+17 \\
+5 \\
+15\end{array}$ & $\begin{array}{l}\omega_{7} \nu_{\mathrm{Cl}, \mathrm{s}} \\
\omega_{9} \nu_{\mathrm{Cl}, \mathrm{a}} \\
\omega_{6} \delta_{\mathrm{H}} \\
\omega_{8} \delta_{\mathrm{H}}\end{array}$ & $\begin{array}{r}+12 \\
+13 \\
+8 \\
+6\end{array}$ \\
\hline \multicolumn{2}{|c|}{$\mathrm{CHClBr}_{2}$} & \multicolumn{2}{|c|}{$\mathrm{CHBr}_{3}$} \\
\hline $\begin{array}{l}\omega_{g} \nu \mathrm{B} r, \mathrm{~B} \\
\omega_{\tau} \nu \mathrm{Cl} \\
\omega_{\delta} \delta \mathrm{H} \\
\omega_{6} \delta \mathrm{H}\end{array}$ & $\begin{array}{r}+14 \\
+12 \\
+7 \\
+5\end{array}$ & $\begin{array}{l}\omega_{68} \delta \delta_{\mathrm{H}} \\
\omega_{1} \nu_{\mathrm{H}}\end{array}$ & $\begin{array}{r}+7 \\
+18\end{array}$ \\
\hline \multicolumn{2}{|c|}{$\mathrm{CH}_{2} \mathrm{Cl}_{2}$} & \multicolumn{2}{|c|}{$\mathrm{CH}_{2} \mathrm{~F}_{2}$} \\
\hline 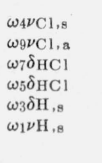 & $\begin{array}{r}+9 \\
+15 \\
0 \\
+4 \\
+38 \\
+14\end{array}$ & \multirow[t]{3}{*}{$\begin{array}{l}\omega_{2} \delta_{\mathrm{F}, \mathrm{s}} \\
\omega_{9} \nu_{\mathrm{F}, \mathrm{s}} \\
\omega_{4} \nu_{\mathrm{F}, \mathrm{s}} \\
\omega_{7} \delta_{\mathrm{HF}} \\
\omega_{8} \delta_{\mathrm{HF}} \\
\omega_{1} \nu_{\mathrm{H}, \mathrm{s}} \\
\omega_{6} \nu_{\mathrm{H}, \mathrm{s}}\end{array}$} & $\begin{array}{r}-3 \\
+35 \\
+38 \\
+6 \\
0 \\
-14 \\
-18\end{array}$ \\
\hline \multicolumn{2}{|c|}{$\mathrm{CHF}_{3}$} & & \\
\hline $\begin{array}{l}\omega_{25} \delta \delta_{\mathrm{F}, \mathrm{a}} \\
\omega_{44} \delta \mathrm{F}, \mathrm{s} \\
\omega_{3} \nu_{\mathrm{F}, \mathrm{s}} \\
\omega_{7} \delta \delta_{\mathrm{F}, \mathrm{a}} \\
\omega_{68} \nu \mathrm{H} \\
\omega_{1} \nu_{\mathrm{H}}\end{array}$ & $\begin{array}{r}+1 \\
+3 \\
+33 \\
-7 \\
-6 \\
-26\end{array}$ & & \\
\hline
\end{tabular}

\subsection{Discussion on Particular Molecules}

It does not seem necessary to discuss all the assignments for each molecule, since in most cases the assignments are in substantial agreement with the work of other investigators who have studied these molecules, either by the infrared or Raman spectra. In a few instances, however, where certain points have been discussed in the literature, it seems desirable to justify our choices further. It should be pointed out that in many cases final decisions can hardly be reached until the rotational fine structure of the bands in question is studied under very high resolution.

\section{6. $\mathrm{CF}_{4}$}

The bands may all be assigned as $\mathrm{F}_{2}$ combinations or overtones of the two infrared active frequencies $629 \mathrm{~cm}^{-1}$ and $1,277 \mathrm{~cm}^{-1}$, and the Raman-active $437 \mathrm{~cm}^{-1}(\mathrm{E})$ and $904 \mathrm{~cm}^{-1}\left(\mathrm{~A}_{1}\right)$. The fact that these pairs are each nearly in the ratio $1: 2$ would make it possible for Fermi resonance to appear in many of the combinations; however, as the observations give a band of high intensity at each position, it appears that the interactions are not strong, and that the resonance is only partial. There is a very weak peak at $\sim 1,238 \mathrm{~cm}^{-1}$, which may correspond to the weaker component of the $2 \nu_{479}, \nu_{168} \mathrm{~F}_{2}$ pair; the corresponding combinations with $\nu_{1}$ also appear at 2,123 and 2,160 with a relative intensity of about 1:5. The unresolved rotational envelopes of the 629 and $1,277 \mathrm{~cm}^{-1}$ fundamentals are quite different, the former showing well separated $P, D$, and $R$ branches, with a $P-R$ distance of $\sim 33 \mathrm{~cm}^{-1}$, whereas in the latter this distance is much less and unresolved. This is due to the interaction with the rotation of angular momentum of vibration, and is in qualitative agreement with calculation: the $\zeta$-factor for the valence vibration $\nu_{168}$ should be about +0.68 and for $\nu_{479}$ about -0.18 , so that the rotational spacings for the two bands should be in the ratio $(0.32 / 1.18) \sim 0.3$. The combination bands of these fundamentals with $\nu_{3}$ show the same behavior; for example, 1,532 is found with a $P-R$ spacing of $\sim 38 \mathrm{~cm}^{-1}$, whereas 2,160 is narrow and unresolved.

\section{7. $\mathrm{CF}_{3} \mathrm{Cl}$}

The two lowest-frequency fundamentals, $\omega_{25}(\mathrm{E})$ and $\omega_{4}\left(\mathbf{A}_{1}\right)$ are very weak in the infrared, but appear strongly in the Raman spectrum of the liquid. Although a weak band appears at $450 \mathrm{~cm}^{-1}$, in the infrared spectrum, it seems preferable to adopt $470 \mathrm{~cm}^{-1}$ of the Raman spectra for the vapor-phase frequency of $\omega_{4}$, as this accounts for the weak absorption at $635 \mathrm{~cm}^{-1}$ as a difference band and gives satisfactory agreement in a number of combinations.

\section{8. $\mathrm{CF}_{2} \mathrm{Cl}_{2}$}

The force-constant calculations definitely require that there be five (deformation) frequencies below $500 \mathrm{~cm}^{-1}$ and only four above $600 \mathrm{~cm}^{-1}$. Hence the assignment of both the strong bands at 882 and $922 \mathrm{~cm}^{-1}$ as fundamentals cannot be correct; it is very reasonable to consider this a case of Fermi resonance, with the original $\omega_{6}$ fundamental $\left(B_{1}\right)$ at $906 \mathrm{~cm}^{-1}$ split by interaction with the combination $\omega_{4}\left(A_{1}\right) 455+\omega_{7}\left(B_{1}\right) 437$. The region from 420 to 480 $\mathrm{cm}^{-1}$ must be studied under higher resolution before the values for the three overlapping frequencies $\omega_{6}$, $\omega_{4}$, and $\omega_{9}$ can be considered certain. The value of $455 \mathrm{~cm}^{-1}$ for $\omega_{4}$ is from the Raman effect.

\section{9. $\mathrm{CFCl}_{3}$}

As in the other chloro-fluoromethanes, the deformation fundamentals are very weak, but Ramaneffect measurements enable a choice to be made between the fundamentals and the equally intense difference bands at wavelengths greater than $20 \mu$. The combination band at $932 \mathrm{~cm}^{-1}$ is more intense than the fundamentals on which it is based. This may result from a partial Fermi resonance with the very intense $\omega_{68}$. The $\nu_{\mathrm{F}}$ frequency shows a $+17 \mathrm{~cm}^{-1}$ shift from the liquid value found in the Raman effect [9].

\subsection{0. $\mathrm{CCl}_{4}$}

This much-studied molecule shows one of the earliest-recognized examples of Fermi resonance in the strong pair at 762 to $785 \mathrm{~cm}^{-1}$. The unsplit position of the $\omega_{168}$ is taken as the exact center, $773 \mathrm{~cm}^{-1}$, since the two bands are nearly of equal intensity, in the liquid. In the vapor the resonance with $319+458$ is less exact, the intensity ratio now being about $4: 1$, the higher frequency component being the stronger. This is in accord with the general observation that the vapor-liquid shift is greatest for the asymmetric valence vibrations. It is of consider- 
able interest that the doubling also appears in two difference bands, arising from $\omega_{25}$ and $\omega_{479}$.

\subsection{1. $\mathrm{CCl}_{3} \mathrm{Br}$ and $\mathrm{CCl}_{2} \mathrm{Br}_{2}$}

In these heavy molecules many fundamentals lie beyond the limit of our observation, but are known from the Raman effect. There is considerable absorption without definite structure beyond $20 \mu$, corresponding to many possible overlapping difference and combination bands of the deformation frequencies.

\subsection{2. $\mathrm{CHF}_{3}$}

This molecule has been studied under very high resolution in the photographic infrared [10]. We have also examined a number of the combination bands under high resolution. The only question in the frequency assignment concerns the value of $\omega_{3}$, the $\nu_{F}$, fundamental. Its intensity and polarization in the Raman spectrum definitely prove that in the liquid it fallo at $1,116 \mathrm{~cm}^{-1}$. In the infrared spectrum of the gas there is no indication of any weak absorption to longer wavelengths of the $1,152 \mathrm{~cm}^{-1}$ peak. It is therefore believed that the $\nu_{F}, \mathrm{~s}$ and $\nu_{F}$, a fundamentals overlap at this point, the difference from the Raman effect being due to the vapor-liquid shift, whereas the weak band at $1,209 \mathrm{~cm}^{-1}$ is a combination tone, A high-resolution study of this region would be of interest. Our force-constant calculations, in agreement with those of Rank, Shull, and Pace, confirm the assignments of $700 \mathrm{~cm}^{-1}$ to $A_{1}$ and $507 \mathrm{~cm}^{-1}$ to $\mathrm{E}$ symmetry, although the rotational envelopes of the two bands are nearly identical.

\subsection{3. $\mathrm{CHF}_{2} \mathrm{Cl}$}

The assignment of the $\omega_{3}\left(\nu_{F},{ }_{\mathrm{s}}\right)$ fundamental differs from that of Glockler, Edgell, and Leader [11] obtained from the Raman effect. There are two medium-intensity bands to choose between, at 833 and $1,178 \mathrm{~cm}^{-1}$. The higher value fits much better with the related molecules. The low frequency, which is fairly strong in the Raman effect, and which may well be accounted for as $2 \times 422 \mathrm{~cm}^{-1}$, undoubtedly gains in intensity by partial Fermi resonance with $\omega_{7}$, at $809 \mathrm{~cm}^{-1}$.

\subsection{4. $\mathrm{CHFCl}_{2}, \mathrm{CHCl}_{3}, \mathrm{CHCl}_{2} \mathrm{Br}, \mathrm{CHClBr}_{2}, \mathrm{CHBr}_{3}$}

In all these molecules, there is little question as to the assignment of fundamentals, when the infrared and Raman results are both considered. All show absorption in the long-wavelength region. The 262 $\mathrm{cm}^{-1}$ fundamental of $\mathrm{CHCl}_{3}$ is relatively strong, but occurs just at the long-wavelength limit of the TlBr-I prism.

\subsection{5. $\mathrm{CHI}_{3}$}

The results for this molecule are quite incomplete. Because of its physical properties (solid at room. temperature and above, subliming with decomposition) it could be studied only in solution. Its chemical instability prevented the use of high concentration, and rapid surveys of the spectrum were required. The Raman frequencies, and rough forceconstant calculations, show that two of the fundamentals lie at very long wavelengths. The few bands observed find a natural interpretation by analogy with the corresponding molecules.

\subsection{6. $\mathrm{CH}_{2} \mathrm{~F}_{2}$}

This molecule has been investigated recently under high resolution [12]. As mentioned above, highresolution studies in the 1.6 to $2.5 \mu$, region have also been made in the radiometry laboratory of the Bureau. The assignment of fundamentals differs from that of the above authors, but is in agreement with the one very recently offered by Rank, Shull, and Pace, [8] and with the force-constant calculations. The Raman-effect data leave no doubt that in the liquid $\omega_{3}$ is at $1,508 \mathrm{~cm}^{-1}$. This $\nu_{H, s}$ frequency might well be very weak in the infrared and escape detection, since it falls in a region where the $\mathrm{H}_{2} \mathrm{O}$ absorption masks weak bands. The band structures found by Stewart and Nielsen are equally well accounted for by the assignment, since the $B_{1}$ combination at $2,945 \mathrm{~cm}^{-1}$ can be ascribed to $\omega_{3}+\omega_{8}$. As mentioned above in connection with table 11 , a relatively large liquid-vapor shift may be invoked to reconcile the Raman and infrared measurements in the region of $1,100 \mathrm{~cm}^{-1}$. The depolarized Raman line at 1,054 $\mathrm{cm}^{-1}$ can be attributed to $\omega_{9}$, rather than to $2 \omega_{4}$ which should be polarized. The $\omega_{5}$ frequency, whose $\mathrm{A}_{2}$ symmetry should make it inactive, appears weakly at $1,262 \mathrm{~cm}^{-1}$, presumably due to Coriolis interaction with $\omega_{7}\left(B_{2}\right)$ at $1,176 \mathrm{~cm}^{-1}$, or to breakdown of the strict selection rules induced by the strong polar intermolecular forces. There are two weak bands, at 816 and $1,349 \mathrm{~cm}^{-1}$ which cannot be assigned. They may be due to impurities.

\subsection{7. $\mathrm{CH}_{2} \mathrm{Cl}_{2}$ and $\mathrm{CH}_{2} \mathrm{I}_{2}$}

These molecules show no unexpected effects. There is a close parallelism among the spectra of all the methylene halides. These will be discussed at greater length in connection with high-resolution studies in the overtone region.

The authors are indebted to N. Acquista and Mary A. Lamb for assistance in the observations of the spectra.

\section{References}

[1] G. Herzberg, Infrared and Raman spectra of polyatomic molecules (D. van Nostrand Co., Inc., New York, N. Y., 1945).

[2] K. W. F. Kohlrausch, Ramanspektren (Becker \& Erler, Leipzig, 1943)

[3] E. K. Plyler, R. Stair, and C. J. Humphreys, J. Research NBS 38, 211 (1947) RP1769.

[4] E. K. Plyler, H. W. Smith, and N. Acquista, J. Research NBS 44, 503 (1950) RP2097.

[5] J. C. Decius, J. Chem. Phys. 16, 214 (1948),

[6] T. Simanouti, J. Chem. Phys. 17, 245 (1949).

[7] E. L. Pace, J. Chem. Phys. 18, 881 (1950).

[8] D. H. Rank, E. R. Shull, and E. L. Pace, J. Chem. Phys. 18, 885 (1950).

[9] J. P. Zietlow, F. F. Cleveland, and A. G. Meister, J. Chem. Phys. 18, 1076 (1950).

[10] H. J. Bernstein and G. Herzberg, J. Chem. Phys. 16, 30 (1948).

[11] G. Glockler, W. F. Edgell, and G. R. Leader, J. Chem. Phys. 9, 224 (1941).

[12] H. B. Stewart and H. H. Nielsen, Phys. Rev. 75, 640 (1949).

Washington, January 16, 1951. 